\title{
Relay Selection Strategies for MIMO Two-Way Relay Networks with Spatial Multiplexing
}

\author{
Shashindra Silva, Student Member, IEEE, Gayan Amarasuriya, Member, IEEE, Chintha Tellambura Fellow, IEEE, \\ Masoud Ardakani, Senior Member, IEEE
}

\begin{abstract}
Relay selection strategies help to improve spectral and energy efficiencies, to enhance transmission robustness, or to reduce latency in multi-relay cooperative networks. Two novel relay selection strategies are proposed and analyzed here for multipleinput multiple-output (MIMO) amplify-and-forward (AF) two-way relay networks (TWRNs) with spatial multiplexing. Specifically, they are designed to maximize the effective end-to-end signal-to-noise ratio (SNR), and thereby minimize the overall outage probability or maximize the achievable sum rate. Interestingly, the first strategy amounts to maximizing the minimum of the eigenvalues of the Wishart matrices from the selected relay to the two user nodes. Counter-intuitively, the latter strategy amounts to maximizing the minimum of the determinant of the same Wishart matrices. The performance of these two strategies is investigated by deriving lower/upper bounds of the overall outage probability and the average sum rate approximations in closed-form. Further, the asymptotic high SNR approximations of the outage probability are derived, and thereby, the achievable diversity-multiplexing trade-off is quantified. This trade-off reveals that whenever the sum of relay antennas is fixed, the achievable diversity order is always a constant, and hence, the multiplexing gain can indeed be improved by equally distributing antennas among the available set of relays. Our results reveal that relay selection indeed significantly alleviates the inherent diversitygain loss associated with the use of available degrees of freedom for spatial multiplexing.
\end{abstract}

Index terms - Relay Networks, MIMO, Spatial Multiplexing

\section{INTRODUCTION}

Wireless relays help to improve spectral and energy efficiency, to enhance transmission robustness, or to reduce latency in multirelay cooperative networks. However, since multiple spatially distributed relays require multiple orthogonal channel-uses whenever the data is transmitted via all available relays for preventing interference, these additional channel-uses indeed degrade the overall spectral efficiency. To prevent this, one or more nodes can be selected from a pool of nodes to act as relays between a transmitter (Tx) and a receiver (Rx) [2]. This process is known as relay selection and has been widely investigated. Performance measures used for relay selection strategies can be spectral and energy efficiency, transmission robustness, or latency in multirelay cooperative networks [3], [4].

However, many studies (e.g, [2]-[4] and references therein) consider one-way relay networks (OWRNs) with unidirectional data-flows employing half-duplex nodes. These require four channel-uses for a bidirectional mutual data exchange between

The authors Shashindra Silva, Chintha Tellambura, and Masoud Ardakani are with the Department of Electrical and Computer Engineering, University of Alberta, Edmonton, AB, Canada T6G 2V4, Phone: (780) 492-7228, Fax: (780) 492-1811 Email: \{jayamuni,chintha,ardakani\}@ece.ualberta.ca.

Gayan Amarasuriya is with the Department of Electrical Engineering, Princeton University, Princeton, NJ, USA, 08540, Email: gbaduge@ princeton.edu

This work in part was presented at the IEEE International Conference on Communications (ICC), London, UK, 2015 [1]. The study presented in this paper is supported by TELUS Communications Company and Natural Sciences and Engineering Research Council of Canada (NSERC). two nodes. In contrast, this exchange is possible with just two channel uses when two-way relay networks (TWRNs) with bidirectional data-flows are employed. Consequently, the spectral efficiency of TWRNs is twice that of OWRNs [5], [6], and thus, they are currently being examined for next generation wireless communication standards, including Long-term evolutionadvanced (LTE-A) [7], [8].

Because these standards demand significant performance improvements in terms of data rates and link-reliability, multipleinput multiple-output (MIMO) wireless technology is essential [9]. Respectively, these improvements emanate from spatial multiplexing gains and diversity gains exploited from rich-scattering wireless channels. Moreover, both spatial multiplexing and diversity benefits can indeed be achieved subjected to a fundamental trade-off whenever efficient space-time code designs are employed [10]. Naturally, the use of MIMO in TWRNs offers performance benefits [6], [11], [12].

It is therefore important to develop relay selection strategies for TWRNs employing MIMO-enabled user and relay nodes [6], [11], [13], [14]. The prototype network we consider here consists of two user nodes and a set of two-way relay nodes (all nodes are MIMO). In this setup, the relay selection task is however complicated by the number of available design choices. First, because we have several performance metrics available, in this paper, we choose to maximize the SNR of the worst data substream at the weakest user node (or equivalently the effective end-to-end signal-to-noise ratio (SNR)), and thereby minimizing the outage probability of the two users, which is equivalently done by maximizing the diversity order of the two user channels. On the other hand, we can also maximize the sum of data rates of the two users. Second, with MIMO, nodes can use various transmit filtering and receive filter techniques. We will only consider zero-forcing (ZF) transmit and receive filtering for the two user nodes. ZF is a classical scheme which has the advantage of decoupling the MIMO channel to multiple substreams but at the cost of noise coloring [9]. Depending on the availability of channel knowledge, other filtering schemes include maximal ratio combining, maximal ratio transmission, eigen-beamforming and others [9], [15]. Although these schemes are not investigated in this paper, they may prove fertile grounds for further research.

Prior related research on MIMO TWRN: In [6] joint relay and antenna selection strategies over Rayleigh fading are investigated, with closed-form exact and high SNR approximations of outage probability. Thus, achievable diversity order is quantified. Further, [6] analyzes and quantifies the performance degradation due to feedback-delay effect and spatially correlated fading. Reference [12] develops a joint beamforming and relay selection strategy to maximize the end-to-end SNR and hence to minimize the overall outage probability. In particular, in [12], the overall outage probability is derived in closed-form, and thereby, the achievable 
diversity order is quantified. Note that the relay selection strategies in [6] and [12] have been developed for a single end-toend spatial data-stream. Thus, relay selection for MIMO TWRNs with multiple end-to-end spatial data-streams (i.e., with spatial multiplexing) has not yet been investigated.

For the sake of completeness, several important prior studies on relay selection for single-antenna TWRNs are next summarized. In [13], single and multiple relay selection schemes are developed and analyzed. Single-relay selection is based on maximizing the worst SNR of the two user nodes. Further, in the multiple relay selection scheme of [13], a subset of available relays are selected by using the concept of relay ordering proposed in [3]. Here, the available relays are first ordered in the ascending order of the endto-end SNR, and then, the best subset of relays, which maximizes the worst SNR of the two user nodes, are selected. Further, in [16], an optimal relay selection scheme is developed with full-duplex nodes based on maximizing the effective signal-to-interferenceplus-noise ratio (SINR). All aforementioned studies [13], [16] treat AF relays. But [17]-[19] consider decode-and-forward (DF) relays. Moreover, in [14], [20], the relay selection schemes are studied for single-antenna TWRNs with physical-layer network coding.

Motivation and our contribution: In the extensive wireless relay literature, only two studies develop relay selection strategies for MIMO TWRNs. Moreover, these strategies in [6] and [12] are limited to a single end-to-end spatial data stream only. Thus, they are more suited for practical scenarios where the corresponding wireless channels are ill-conditioned (due to lack of rich scattering) or if the transmission reliability via the diversity gains is preferred over the data rates via spatial multiplexing gains. In this context, no prior results exist on designing and analyzing of relay selection strategies for MIMO TWRNs with spatial multiplexing (i.e., with more than one end-to-end spatial data-streams). This observation thus motivates our work and fills the aforementioned gap in relay literature by proposing and analysing two novel relay selection strategies for MIMO TWRNs with spatial multiplexing. Our strategies are motivated by the practical need for boosting the throughput of MIMO TWRNs operating in rich-scattering wireless channels, and hence prioritize the use of available degrees of freedom for spatial multiplexing. The proposed relay selection strategies and our main contributions can be next summarized as follows:

Specifically, our first relay selection strategy maximizes the SNR of the worst data substream at the weakest user node and consequently minimizing the overall outage probability. Interestingly, it amounts to selecting the relay which maximizes the minimum of the eigenvalues of the Wishart matrices of the selected relay to the two user nodes. Moreover, our second strategy maximizes the achievable sum rate. Unlike the first strategy, this one selects the relay which maximizes the minimum of the determinants of the same Wishart matrices. Intuitively, the first strategy is designed to maximize the overall diversity order by alleviating the reduction of the available degrees of freedom due to spatial multiplexing, otherwise majority of the achievable degrees of freedom would be utilized for boosting the diversity order as shown in [6], [12]. Counter-intuitively, the second one maximizes the overall spatial multiplexing gain by maximizing the effective determinant of the corresponding Wishart matrices, and thereby paving the way to transmit multiple data-streams through well-conditioned spatial sub-channels.

The performance of the two proposed relay selection strategies is investigated by deriving the lower/upper bounds of the overall outage probability and the average sum rate approximations in closed-form. Further, the asymptotic high SNR outage probability approximations are then used for deriving the achievable diversity-multiplexing trade-off. Thereby, the maximum overall diversity order and achievable spatial multiplexing gain are quantified to obtain valuable insights into practical implementation of relay selection strategies for MIMO TWRNs. Moreover, rigorous simulation results are presented to investigate/compare the performance of the proposed selection strategies and as well to verify our analysis.

Notation: $\mathbf{Z}^{H},[\mathbf{Z}]_{k}$, and $\lambda_{k}\{\mathbf{Z}\}$ denote the Hermitian-transpose, the $k$ th diagonal element, and the $k$ th eigenvalue of the matrix, $\mathbf{Z}$, respectively. $\mathcal{E}_{\Lambda}\{z\}$ is the expected value of $z$ over $\Lambda$, and the operator $\otimes$ denotes the Kronecker product. $\mathbf{I}_{M}$ and $\mathbf{O}_{M \times N}$ are the $M \times M$ Identity matrix and $M \times N$ matrix of all zeros, respectively. $f(x)=o(g(x)), g(x)>0$ states that $f(x) / g(x) \rightarrow$ 0 as $x \rightarrow 0$. Further, $E_{1}(z)$ is the exponential integral function for the positive values of the real part of $\mathrm{z}$ [21, Eqn. (8.211)]. $\Gamma(z)$ is the Gamma function [21, Eqn. (8.310.1)], and $\Gamma(a, z)$ is the upper incomplete Gamma function [21, Eqn. (8.350.2)].

\section{MIMO ZF TWRN SYSTEMS}

This section presents the system, channel, and signal models. Further, the exact end-to-end SNRs at the user nodes are derived, leading to the lower and upper bounds of the SNRs.

\section{A. System and channel model}

The system model consists of two user nodes $\left(U_{1}\right.$ and $\left.U_{2}\right)$ and $L$ relay nodes $\left(R_{l}\right.$ for $\left.l \in\{1, \cdots, L\}\right)$. User node $U_{i}$ is equipped with $N_{i}$ antennas for $i \in\{1,2\}$, and the $l$ th relay node has $N_{R_{l}}$ antennas. All nodes are assumed half-duplex terminals, and all channel amplitudes are assumed independently distributed, frequency flat-Rayleigh fading. Thus, the channel matrix from $U_{i}$ to $R_{l}$ can be defined as $\mathbf{F}_{i, l} \sim \mathcal{C} \mathcal{N}_{N_{R_{l}} \times N_{i}}\left(\mathbf{0}_{N_{R_{l}} \times N_{i}}, \mathbf{I}_{N_{R_{l}}} \otimes \mathbf{I}_{N_{i}}\right)$, where $i \in\{1,2\}$ and $l \in\{1, \cdots, L\}$. The channel coefficients are assumed to be fixed during two consecutive time-slots, and hence, the channel matrix from $R_{l}$ to $U_{i}$ can be written as $\mathbf{F}_{l, i}=\mathbf{F}_{i, l}^{T}$ by using the reciprocity property of wireless channels. The additive noise at all the receivers is modelled as complex zero mean additive white Gaussian (AWGN) noise. The direct channel between $U_{1}$ and $U_{2}$ is assumed to be unavailable due to large pathloss and heavy shadowing effects [5], [11], [22].

One complete transmission cycle of our two-way relaying model consists of two phases, namely (i) multiple-access (MAC) phase and (ii) broadcast (BC) phase. In the MAC phase, two user nodes utilize transmit-ZF precoding for transmitting their signals simultaneously towards the selected relay. Then, the relay receives a superimposed signal which is a function of both signals belonging to two users. During the BC phase, the selected relay transmits an amplified version of its received signal back to two users. Then, each user node employs its corresponding receive-ZF detector to receive the signals belonging to the other user node by using self-interference cancellation [5]. 
It is worth noting that for employing transmit-ZF, the number of antennas at the transmitter should be equal for higher than that of the receiver [9, pp. 210]. Similarly, in order to use receive$\mathrm{ZF}$, the antenna count at the receiver should be equal or higher than that of the transmitter [9, pp. 153]. To fulfil the aforementioned requirement, the antenna configurations at the user nodes and relays should satisfy the following antenna constraint: $N_{1} \geq \max _{l \in\{1, \cdots, L\}} N_{R_{l}}$ and $N_{2} \geq \max _{l \in\{1, \cdots, L\}} N_{R_{l}} \rightarrow$ $\min \left(N_{1}, N_{2}\right) \geq \max _{l \in\{1, \cdots, L\}} N_{R_{l}}$.

Employing multiple-antennas at the user nodes has been one of the limitations for ubiquitous usage of MIMO technology in the current wireless systems. However, the recent research developments in millimeter wave (mmWave) wireless communications render MIMO practically viable even at the user nodes because of the extremely short wavelengths associated with the mmWave frequency bands such as $28 \mathrm{GHz}$ and $38 \mathrm{GHz}$ [23]. Consequently, mmWave frequency bands can be exploited to design MIMO transceivers and other RF elements with much smaller physical dimensions [24]. Thus, employing MIMO even at the user nodes will be practically viable in the next generation wireless standards, and to this end, our system model would be useful in practice.

\section{B. Signal model}

During two time-slots, $U_{1}$ and $U_{2}$ exchange their signal vectors $\left(\mathbf{x}_{1}\right.$ and $\left.\mathbf{x}_{2}\right)$ by selecting one of the available $L$ relays. Here, the selected relay is denoted as $R_{l}$ for the sake of the exposition. In the first time-slot, $U_{1}$ and $U_{2}$ transmit $\mathbf{x}_{1}$ and $\mathbf{x}_{2}$, respectively, towards $R_{l}$ by employing transmit-ZF precoding over the multiple access channel. The received signal at $R_{l}$ can then be written as

$$
\mathbf{y}_{R_{l}}=g_{1, l} \mathbf{F}_{1, l} \mathbf{W}_{T_{1, l}} \mathbf{x}_{1}+g_{2, l} \mathbf{F}_{2, l} \mathbf{W}_{T_{2, l}} \mathbf{x}_{2}+\mathbf{n}_{R_{l}},
$$

where $l \in\{1, \cdots L\}$ and the $N_{R_{l}} \times 1$ signal vector $\mathbf{x}_{i}$ satisfies $\mathcal{E}\left[\mathbf{x}_{i} \mathbf{x}_{i}^{H}\right]=\mathbf{I}_{N_{R_{l}}}$ for $i \in\{1,2\}$. Thus, the $N_{i} \times 1$ precodedtransmit signal vector at $U_{i}$ is given by $\mathbf{W}_{T_{i, l}} \mathbf{x}_{i}$. In $(1), \mathbf{n}_{R_{l}}$ is the $N_{R_{l}} \times 1$ zero mean AWGN vector at $R_{l}$ satisfying $\mathcal{E}\left(\mathbf{n}_{R_{l}} \mathbf{n}_{R_{l}}^{H}\right)=$ $\mathbf{I}_{N_{R_{l}}} \sigma_{R_{l}}^{2}$. In particular, in (1), $\mathbf{W}_{T_{i, l}}$ is the transmit-ZF precoder at $U_{i}$ and can be defined as [25]

$$
\mathbf{W}_{T_{i, l}}=\mathbf{F}_{i, l}^{H}\left(\mathbf{F}_{i, l} \mathbf{F}_{i, l}^{H}\right)^{-1} \text { for } i \in\{1,2\} \text { and } l \in\{1, \cdots, L\} .
$$

Moreover, in (1), $g_{i, l}$ is the power normalizing factor at $U_{i}$ and is designed to constrain its long-term transmit power as follows [11]:

$g_{i, l}=\sqrt{\frac{\mathcal{P}_{i}}{\mathcal{E}\left[\operatorname{Tr}\left(\mathbf{W}_{T_{i, l}} \mathbf{W}_{T_{i, l}}^{H}\right)\right]}}=\sqrt{\frac{\mathcal{P}_{i}}{\mathcal{E}\left[\operatorname{Tr}\left(\left(\mathbf{F}_{i, l} \mathbf{F}_{i, l}^{H}\right)^{-1}\right)\right]}}$,

where $i \in\{1,2\}, l \in\{1, \cdots, L\}$, and $\mathcal{P}_{i}$ is the transmit power at $U_{i}$. Next, the expected value of the trace of an inverse Wishart matrix can be evaluated as [26]

$$
\operatorname{Tr}\left(\mathcal{E}\left[\left(\mathbf{F}_{i, l} \mathbf{F}_{i, l}^{H}\right)^{-1}\right]\right)=\frac{N_{R, l}}{N_{i}-N_{R, l}} .
$$

Next, by substituting (4) into (3), the power normalizing factor at $U_{i}$ can be derived as

$$
g_{i, l}=\sqrt{\frac{\mathcal{P}_{i}\left(N_{i}-N_{R_{l}}\right)}{N_{R_{l}}}} \text { for } i \in\{1,2\} \text { and } l \in\{1, \cdots, L\} .
$$

In order to evaluate (4) finitely and hence to obtain a finite nonzero value for $g_{i, l}(5)$ at each user node, the constraint $N_{i} \neq$ $N_{R_{l}}$ needs to be satisfied. Thus, in order to employ transmit$\mathrm{ZF} /$ receive-ZF at $U_{1}$ and $U_{2}$ during two transmission phases, and for satisfying the long-term transmit power constraint at $R_{l}$, the antenna configurations at the relay and user nodes should satisfy the condition $\min \left(N_{1}, N_{2}\right)>\max _{l \in\{1, \cdots, L\}} N_{R_{l}}$.

In the second time-slot, $R_{l}$ amplifies $\mathbf{y}_{R_{l}}$ and broadcasts this amplified-signal towards both user nodes. Each user node then receives its signal vector by using the corresponding receive-ZF detector as

$$
\mathbf{y}_{U_{i, l}}=\mathbf{W}_{R_{i, l}}\left(G_{l} \mathbf{F}_{l, i} \mathbf{y}_{R_{l}}+\mathbf{n}_{i}\right),
$$

where $i \in\{1,2\}$ and $l \in\{1, \cdots, L\}$. Further, $G_{l}$ is the amplification factor at $G_{l}$ and can be defined as

$$
G_{l}=\sqrt{\frac{\mathcal{P}_{R_{l}}}{g_{1, l}^{2}+g_{2, l}^{2}+\sigma_{R_{l}}^{2}}},
$$

where $g_{l, i}$ is defined in (5) and $\sigma_{R_{l}}^{2}$ is the variance of the additive noise at $R_{l}$. Moreover, in (6), $\mathcal{P}_{R_{l}}$ is the transmit power at $R_{l}$, $\mathbf{F}_{l, i}=\mathbf{F}_{i, l}^{T}$, and $\mathbf{n}_{i}$ is the $N_{i} \times 1$ zero mean AWGN at $U_{i}$ satisfying $\mathcal{E}\left(\mathbf{n}_{i} \mathbf{n}_{i}^{H}\right)=\mathbf{I}_{N_{i}} \sigma_{i}^{2}$ for $i \in\{1,2\}$. Furthermore, in (6), $\mathbf{W}_{R_{i, l}}$ is the receive-ZF matrix at $U_{i}$, and can be given as [25]

$\mathbf{W}_{R_{i, l}}=\left(\mathbf{F}_{l, i}^{H} \mathbf{F}_{l, i}\right)^{-1} \mathbf{F}_{l, i}^{H}$ for $i \in\{1,2\}$ and $l \in\{1, \cdots, L\}$.

Remark II.1: In our system model, the two user nodes use simple transmit-ZF and receive-ZF filtering. Thus, the resulting ZF precoder/detector have closed-form solutions (based on generalized inverse) unlike the iterative solution needed for the optimal precoder/detectors in [15]. Further, the authors in [15] also show that $\mathrm{ZF}$ precoders and detectors can provide a significant fraction of the performance gains achieved by the optimal counterparts. These reasons motivate the investigation of $\mathrm{ZF}$ transmit and receive filtering.

\section{Exact end-to-end SNR}

In this subsection, the exact end-to-end SNR of the $k$ th data substream for $k \in\left\{1, \cdots, N_{R_{l}}\right\}$ is derived by using the signalling model presented in Section II-B. To this end, by substituting (1), (2), and (8) into (6), the received signal vector at $U_{i}$ can be written in an alternative form as follows:

$$
\mathbf{y}_{U_{i, l}}=G_{l}\left(g_{i, l} \mathbf{x}_{i}+g_{i^{\prime}, l} \mathbf{x}_{i^{\prime}}+\mathbf{n}_{R_{l}}\right)+\left[\left(\mathbf{F}_{l, i}^{H} \mathbf{F}_{l, i}\right)^{-1} \mathbf{F}_{l, i}^{H}\right] \mathbf{n}_{i},
$$

where $i \in\{1,2\}, i^{\prime} \in\{1,2\}$, and $i \neq i^{\prime}$. By carefully observing the received signal at the user nodes in (9), it can readily be seen that the effective noise term is coloured due to the application of the receive- $\mathrm{ZF}$ detector $\left(\mathbf{W}_{R_{i, l}}\right)$. It is worth noting that this coloured noise resulted due to the fact that the receive-ZF detector matrix is not a unitary matrix. Next, the filtered, coloured noise at the user nodes can explicitly be expressed as follows:

$$
\tilde{\mathbf{n}}_{i}=\left[\left(\mathbf{F}_{l, i}^{H} \mathbf{F}_{l, i}\right)^{-1} \mathbf{F}_{l, i}^{H}\right] \mathbf{n}_{i} .
$$

Next, by using self-interference cancellation to (9), the signal vector of $U_{i^{\prime}}$ received at $U_{i}$ can be extracted as follows:

$$
\tilde{\mathbf{y}}_{U_{i, l}}=G_{l}\left(g_{i^{\prime}, l} \mathbf{x}_{i^{\prime}}+\mathbf{n}_{R_{l}}\right)+\tilde{\mathbf{n}}_{i},
$$




$$
\gamma_{U_{i, l}^{(k)}}=\frac{\left(N_{i^{\prime}}-N_{R_{l}}\right) \bar{\gamma}_{i^{\prime}, l} \bar{\gamma}_{l, i}}{N_{R_{l}} \bar{\gamma}_{l, i}+\left(\left(N_{i^{\prime}}-N_{R_{l}}\right) \bar{\gamma}_{i^{\prime}, l}+\left(N_{i}-N_{R_{l}}\right) \bar{\gamma}_{i, l}+N_{R_{l}}\right)\left[\left(\mathbf{F}_{l, i}^{H} \mathbf{F}_{l, i}\right)^{-1}\right]_{k, k}} .
$$

where $i \in\{1,2\}, i^{\prime} \in\{1,2\}$, and $i \neq i^{\prime}$. By using (11), the post-processing end-to-end SNR of the $k$ th data substream at $U_{i}$ can be derived as

$$
\gamma_{U_{i, l}^{(k)}}=\frac{G_{l}^{2} g_{i^{\prime}, l}^{2}}{G_{l}^{2} \sigma_{R_{l}}^{2}+\sigma_{i}^{2}\left[\left(\mathbf{F}_{l, i}^{H} \mathbf{F}_{l, i}\right)^{-1}\right]_{k, k}} \text { for } k \in\left\{1, \cdots, N_{R_{l}}\right\} \text {. }
$$

By substituting $G_{l}$ (7) and $g_{i^{\prime}, l}$ (5) into (12), the end-to-end SNR in (12) can be written in a more insightful form as shown in (13). Here in (13), $k \in\left\{1, \cdots, N_{R_{l}}\right\}, \bar{\gamma}_{i, l} \triangleq \mathcal{P}_{i} / \sigma_{R_{l}}^{2}, \bar{\gamma}_{l, i} \triangleq \mathcal{P}_{R_{l}} / \sigma_{i}^{2}$, $i \in\{1,2\}, i^{\prime} \in\{1,2\}, l \in\{1, \cdots, L\}$ and $i \neq i^{\prime}$.

Remark II.2: It is worth noting that $\gamma_{U_{1, l}^{(k)}}$ and $\gamma_{U_{2, l}^{(k)}}$ for $k \in$ $\left\{1, \cdots, N_{R_{l}}\right\}$ of (13) are statistically independent for a given $k$ and $l$. However, post-processing SNRs of multiple substreams belonging to a given user node are correlated, i.e., $\gamma_{U_{i, l}^{(k)}}$ and $\gamma_{U_{i, l}^{\left(k^{\prime}\right)}}$ are correlated for a given $i$ and $l$. Due to this correlation effect, derivation of the probability distributions of the SNR of the smallest data substream appears mathematically intractable, and hence, the SNR bounds are derived in the next subsection.

\section{Bounds on the SNR of the smallest data substream and their probability distributions}

In this subsection, lower and upper bounds on the end-to-end SNR of the smallest data substream are derived. In particular, these SNR lower and upper bounds are used in deriving the important performance metrics in closed-form in the sequel.

1) Lower bound on the SNR of the smallest data substream: A lower bound of the SNR of the smallest data substream can be derived as follows: The maximum diagonal element of $\left(\mathbf{F}_{l, i}^{H} \mathbf{F}_{l, i}\right)^{-1}$ can be upper bounded as [25]

$$
\begin{aligned}
\max _{k \in\left\{1 \cdots N_{R_{l}}\right\}}\left[\left(\mathbf{F}_{l, i}^{H} \mathbf{F}_{l, i}\right)^{-1}\right]_{k, k} & \leq \lambda_{\max }\left\{\left(\mathbf{F}_{l, i}^{H} \mathbf{F}_{l, i}\right)^{-1}\right\} \\
& =\lambda_{\min }^{-1}\left\{\mathbf{F}_{l, i}^{H} \mathbf{F}_{l, i}\right\} .
\end{aligned}
$$

By substituting (14) into (13), the SNR of the smallest substream of $U_{i}$ for $i \in\{1,2\}$ can then be lower bounded as follows [11]:

$$
\gamma_{U_{i, l}^{\min }}=\min _{k \in\left\{1 \cdots N_{R_{l}}\right\}} \gamma_{U_{i, l}^{(k)}} \geq \gamma_{U_{i, l}^{\min }}^{\mathrm{lb}}=\frac{\alpha_{i, l}}{\beta_{i, l}+\zeta_{i, l} \lambda_{\min }^{-1}\left\{\mathbf{F}_{l, i}^{H} \mathbf{F}_{l, i}\right\}},
$$

where $\alpha_{i, l}=\left(N_{i^{\prime}}-N_{R_{l}}\right) \bar{\gamma}_{i^{\prime}, l} \bar{\gamma}_{l, i}, \beta_{i, l}=N_{R_{l}} \bar{\gamma}_{l, i}$, and $\zeta_{i, l}=$ $\left(N_{i^{\prime}}-N_{R_{l}}\right) \bar{\gamma}_{i^{\prime}, l}+\left(N_{i}-N_{R_{l}}\right) \bar{\gamma}_{i, l}+N_{R_{l}}$ for $i \in\{1,2\}, i^{\prime} \in\{1,2\}$, $i \neq i^{\prime}$, and $l \in\{1, \cdots, L\}$. It is worth noting that $\gamma_{U_{1, l}^{\min }}^{\mathrm{lb}}$ and $\gamma_{U_{2 l}^{\min }}^{\mathrm{lb}}$ are statistically independent for any $l$.

The bound in (15) is a function of the minimum eigenvalue of Wishart matrix $\mathbf{F}_{l, i}^{H} \mathbf{F}_{l, i}$. The distribution of such eigenvalues is available in the existing literature [27] and using this, the cumulative distribution function $(\mathrm{CDF})$ of $\gamma_{U_{i, l}^{\min }}^{\mathrm{lb}}$ can be written as follows (see Appendix A for the proof):

$F_{\gamma_{U_{i, l}^{\min }}^{\mathrm{lb}}}(x)=\left\{\begin{array}{lc}1-\frac{\operatorname{det}\left[\mathbf{Q}_{i}\left(\frac{\zeta_{i, l} x}{\alpha_{i, l}-\beta_{i, l} x}\right)\right]}{\prod_{j=1}^{N_{R}}\left[\Gamma\left(N_{i}-j+1\right) \Gamma\left(N_{R_{l}}-j+1\right)\right]}, & 0<x<\frac{\alpha_{i, l}}{\beta_{i, l}} \\ 1, & x \geq \frac{\alpha_{i, l}}{\beta_{i, l}} .\end{array}\right.$
In (16), $\mathbf{Q}_{i}(x)$ is an $N_{R_{l}} \times N_{R_{l}}$ matrix with the $(u, v)$ th element given by [27, Eq. (2.73)]

$$
\left[\mathbf{Q}_{i}(x)\right]_{u, v}=\Gamma\left(N_{i}-N_{R_{l}}+u+v-1, x\right) .
$$

2) Upper bound on the SNR of the smallest data substream: Next, an upper bound on the SNR of the smallest data substream can be derived as follows: The maximum diagonal element of $\left(\mathbf{F}_{l, i}^{H} \mathbf{F}_{l, i}\right)^{-1}$ can be lower bounded by any of its diagonal elements [11]. Thus, the SNR of the smallest substream of $U_{i}$ for $i \in\{1,2\}$ can be upper bounded as

$$
\begin{aligned}
\gamma_{U_{i, l}^{\min }} & =\min _{k \in\left\{1 \cdots N_{R_{l}}\right\}} \gamma_{U_{i, l}^{(k)}} \\
& \leq \gamma_{U_{i, l}^{\min }}^{\mathrm{ub}}=\frac{\alpha_{i, l}}{\beta_{i, l}+\zeta_{i, l}\left[\left(\mathbf{F}_{l, i}^{H} \mathbf{F}_{l, i}\right)^{-1}\right]_{k, k}},
\end{aligned}
$$

where $\alpha_{i, l}, \beta_{i, l}$ and $\zeta_{i, l}$ are defined in (15). Again, $\gamma_{U_{1, l}^{\min }}^{\mathrm{ub}}$ and $\gamma_{U_{2, l}^{\min }}^{\mathrm{ub}}$ are statistically independent for any $l$.

Using distribution of the $k$ th diagonal element of the inverse Wishart matrix [28], the CDF of $\gamma_{U_{i, l}^{\min }}^{\mathrm{ub}}$ can then be written as follows (see Appendix A for the proof):

$$
F_{\gamma_{U_{i, l}^{\min }}^{\mathrm{ub}}}(x)= \begin{cases}1-\frac{\Gamma\left(N_{i}-N_{R_{l}}+1, \frac{\zeta_{i, l} x}{\alpha_{i, l}-\beta_{i, l} x}\right)}{\Gamma\left(N_{i}-N_{R_{l}}+1\right)}, & 0<x<\frac{\alpha_{i, l}}{\beta_{i, l}} \\ 1, & x \geq \frac{\alpha_{i, l}}{\beta_{i, l}} .\end{cases}
$$

It is worth noting that both lower and upper SNR bounds in (15) and (18), respectively, converges to the exact SNR whenever $N_{R_{l}}=1$ for $l \in\{1, \cdots, L\}$. Moreover, our analytical and simulation results clearly reveal that the outage bounds derived by using these SNR bounds are asymptotically parallel to the exact outage curves (see Fig. 1) in Section V. Further, the tightness of them significantly improves as $N_{R_{l}}$ approaches unity.

\section{Problem Formulation}

This section formulates two relay selection strategies for MIMO ZF TWRNs. The first strategy (named SS-1) maximizes the SNR of the worst data substream at the weakest user node, and thereby minimizing the overall outage probability. The second one (named SS-2) maximizes the achievable sum rate.

\section{A. Relay selection based on maximizing the effective end-to-end $\operatorname{SNR}(S S-1)$}

In this subsection, the best relay selection strategy is formulated for maximizing the SNR of the worst data substream at the weakest user node, and thereby, for minimizing the overall outage probability. Moreover, if the user nodes are MIMO-enabled with spatial multiplexing, the overall system performance can be improved by selecting a relay which maximizes the SNR of the worst data substream at the weakest user node. Thus, our first 
relay selection strategy can be formulated for maximizing the effective end-to-end SNR as follows:

$$
L^{*}=\underset{l \in\{1, \cdots, L\}}{\operatorname{argmax}}\left[\min \left(\min _{k \in\left\{1, \ldots, N_{R_{l}}\right\}} \gamma_{U_{1, l}^{(k)},} \min _{k \in\left\{1, \ldots, N_{R_{l}}\right\}} \gamma_{U_{2,1}^{(k)}}\right)\right] \text {, }
$$

where $L^{*}$ is the index of the selected relay based on maximizing the SNR of the worst data substream at the weakest user node. Further, in (20), $\gamma_{U_{i, l}^{(k)}}$ is the end-to-end SNR of the $k$ th data substream at $U_{i}$ for $i \in\{1,2\}$ (13).

Next, the overall outage probability of our system set-up is defined as the probability that the effective end-to-end SNR fall below a predefined thershold SNR $\gamma_{t h}$. Thus, the overall outage probability, when the $l$ th arbitrary relay is selected, can be written as follows [11]:

$P_{\text {out }, l}=\operatorname{Pr}\left[\min \left(\min _{k \in\left\{1 \cdots N_{R_{l}}\right\}} \gamma_{U_{1, l}^{(k)}}, \min _{k \in\left\{1 \cdots N_{R_{l}}\right\}} \gamma_{U_{2, l}^{(k)}}\right) \leq \gamma_{t h}\right]$,

where $l \in\{1, \cdots, L\}, \gamma_{t h}$ is the preset threshold SNR, and $\gamma_{U_{i, l}^{(k)}}$ is the end-to-end SNR of the $k$ th data substream at $U_{i}$ for $i \in$ $\{1,2\}$ (13) when the $l$ th relay is selected. Whenever, the best relay is selected based on the selection criterion in (20), it can readily be seen from (21) that the overall outage probability can be minimized.

If the transmit and noise powers at all the relays are the same (i.e., $\mathcal{P}_{R_{l}}=\mathcal{P}_{R}$ and $\sigma_{R_{l}}^{2}=\sigma_{R}^{2}$ for $l \in\{1, \cdots, L\}$ ) and the number of antennas are equal for each relay, then by using (14), the relay selection strategy in (20) can be further simplified as follows:

$$
L^{*}=\underset{l \in\{1, \cdots, L\}}{\operatorname{argmax}}\left[\min \left(\lambda_{\min }\left\{\mathbf{F}_{l, 1}^{H} \mathbf{F}_{l, 1}\right\}, \lambda_{\min }\left\{\mathbf{F}_{l, 2}^{H} \mathbf{F}_{l, 2}\right\}\right)\right] .
$$

Interestingly, as per (22), the best relay, which maximizes the SNR of the worst data substream at the weakest user node, maximizes the minimum of the two smallest eigenvalues of the two Wishart matrices pertinent to the corresponding relay to its two user nodes.

\section{B. Relay selection based on maximizing the sum rate (SS-2)}

This subsection formulates the best relay selection strategy based on maximizing the sum rate. In MIMO TWRNs with symmetric data traffic, each user node needs to transmit its data substreams with a common rate such that these data substreams can be decoded correctly by the intended receivers, and hence, the corresponding sum rate, when the $l$ th relay is selected, can be defined as follows:

$$
\mathcal{R}_{l}=2 \min \left(\mathcal{R}_{U_{1, l}}, \mathcal{R}_{U_{2, l}}\right),
$$

where $\mathcal{R}_{U_{i, l}}$ is the sum of data substreams rates at $U_{i}$ for $i \in$ $\{1,2\}$, and can be written as

$$
\mathcal{R}_{U_{i, l}}=\frac{1}{2} \sum_{k=1}^{N_{R_{l}}} \log \left(1+\gamma_{U_{i, l}^{(k)}}\right) \text {. }
$$

Please note that the factor of two appears in (23) is due to the presence of two user nodes in the TWRN of interest. Further, the pre-log factor of one-half in (24) is due to the two time-slots used for multiple-access and broadcast phases. By first substituting (24) into (23), and then by performing several manipulations, the sum rate can be written in an alternative form as follows:

$$
\mathcal{R}_{l}=\log \left(\min \left(\prod_{k=1}^{N_{R_{l}}}\left(1+\gamma_{U_{1, l}^{(k)}}\right), \prod_{k=1}^{N_{R_{l}}}\left(1+\gamma_{U_{2, l}^{(k)}}\right)\right)\right)
$$

By using (25), the best relay selection based on maximizing the sum rate can then be formulated for moderate-to-high SNR regime as follows: ${ }^{1}$

$$
L^{*} \underset{l \in\{1, \cdots, L\}}{\operatorname{argmax}}\left[\mathcal{R}_{l}\right]=\underset{l \in\{1, \cdots, L\}}{\operatorname{argmax}}\left[\min \left(\prod_{k=1}^{N_{R_{l}}} \gamma_{U_{1, l}^{(k)}}, \prod_{k=1}^{N_{R_{l}}} \gamma_{U_{2, l}^{(k)}}\right)\right],
$$

where $L^{*}$ is the index of the selected best relay. Again, if $\mathcal{P}_{R_{1}}=$ $\mathcal{P}_{R}$ and $\sigma_{R_{1}}^{2}=\sigma_{R}^{2}$ for $l \in\{1, \cdots, L\}$ and number of antennas are equal for each relay, then the relay selection strategy given in (26) can further be simplified as follows:

$$
\begin{aligned}
L^{*}=\underset{l \in\{1, \cdots, L\}}{\operatorname{argmax}}[\min & \left(\prod_{k=1}^{N_{R_{l}}}\left(\left[\left(\mathbf{F}_{l, 1}^{H} \mathbf{F}_{l, 1}\right)^{-1}\right]_{k, k}\right)^{-1},\right. \\
& \left.\left.\prod_{k=1}^{N_{R_{l}}}\left(\left[\left(\mathbf{F}_{l, 2}^{H} \mathbf{F}_{l, 2}\right)^{-1}\right]_{k, k}\right)^{-1}\right)\right] .
\end{aligned}
$$

Then, we recall the inequality between the product of diagonal elements a positive-definite matrix and its determinant as follows [29], [30]:

$$
\operatorname{det}(\mathbf{A}) \leq \prod_{i=1}^{M} a_{i i}
$$

where $\mathbf{A}$ is an $M \times M$ positive-definite matrix with the $i$ th diagonal element denoted as $a_{i i}$ for $i \in\{1, \cdots, M\}$. By using the bound given by (28) in (27), the selection strategy of (26) can further be simplified as

$$
L^{*}=\underset{l \in\{1, \cdots, L\}}{\operatorname{argmax}}\left[\min \left(\operatorname{det}\left(\mathbf{F}_{l, 1}^{H} \mathbf{F}_{l, 1}\right), \operatorname{det}\left(\mathbf{F}_{l, 2}^{H} \mathbf{F}_{l, 2}\right)\right)\right] .
$$

Thus, as per (29), the best relay, which maximizes the sum rate, can be selected by maximizing the minimum of the determinants of the two Wishart matrices pertinent to the corresponding relay to its two user nodes. Our extensive simulations has shown that, the selection of relays based on (29), instead of the real value (27), does not change the maximum sum-rate achieved.

\section{Performance Analysis}

In this section, we derive the performance of our proposed relay selection strategies. For the first selection strategy (SS-1), we first derive the upper and lower bounds of the overall outage probability, and quantify the achievable diversity-multiplexing trade-off by using the corresponding high SNR outage approximations. For the second selection strategy (SS-2), a tight approximation for the achievable ergodic sum rate is derived in closed-form.

\footnotetext{
${ }^{1}$ Eqn. (26) follows due to the fact that for moderate-to-high SNR regime (i.e., for $\left.\gamma_{U_{i, l}^{(k)}}>>1\right)$, the term $\left(1+\gamma_{U_{i, l}^{(k)}}\right)$ can be approximated by $\gamma_{U_{i, l}^{(k)}}$. Thus, we use the approximation $1+\gamma_{U_{i, l}^{(k)}} \stackrel{\approx}{\approx} \gamma_{U_{i, l}^{(k)}}$ for obtaining (26) from (25).
} 


\section{A. Overall outage probability analysis of SS-1}

The overall outage probability of the optimal relay selection based on maximizing the effective end-to-end SNR (SS-1) can be written as follows:

$P_{\text {out }}=\operatorname{Pr}\left[\max _{l \in\{1, \cdots, L\}}\left(\min \left(\left[\gamma_{U_{1, l}^{(k)}}\right]_{\min },\left[\gamma_{U_{2, l}^{(k)}}\right]_{\min }\right)\right) \leq \gamma_{t h}\right],(30$

where $\left[\gamma_{U_{1, l}^{(k)}}\right]_{\min }$ and $\left[\gamma_{U_{2, l}^{(k)}}\right]_{\min }$ are defined as

$$
\begin{aligned}
& {\left[\gamma_{U_{1, l}^{(k)}}\right]_{\text {min }}=\min _{k \in\left\{1 \cdots N_{R_{l}}\right\}} \gamma_{U_{1, l}^{(k)}},} \\
& {\left[\gamma_{U_{2, l}^{(k)}}\right]_{\text {min }}=\min _{k \in\left\{1 \cdots N_{R_{l}}\right\}} \gamma_{U_{2, l}^{(k)}} .}
\end{aligned}
$$

In (30a), $\gamma_{U_{i, l}^{(k)}}$ is the end-to-end SNR of the $k \mathrm{~h}$ data substream at $U_{i}$ received via the $l$ th relay (13).

The closed-form derivation of the exact overall outage probability of (30a) appears mathematically intractable due to the statistical dependence of the substream SNRs of a given user node (see remark II.1). Thus, the upper and lower bounds of the overall outage probability are derived in closed-form.

1) Upper bound on the overall outage probability: An upper bound of the overall outage probability can be written by using the SNR lower bound in (15) as follows 2 :

$$
P_{\text {out }}^{\mathrm{ub}}=\operatorname{Pr}\left[\max _{l \in\{1, \cdots, L\}}\left(\min \left(\gamma_{U_{1, l}^{\min }}^{\mathrm{lb}}, \gamma_{U_{2, l}^{\min }}^{\mathrm{lb}}\right)\right) \leq \gamma_{t h}\right],
$$

where $\gamma_{U_{i, l}^{\min }}^{\mathrm{lb}}$ is a lower bound for the smallest data substream SNR at $U_{i}$ received via the $l$ th relay and is defined in (15). Using the $\mathrm{CDF}$ of a maximum value of a given random variables, the outage upper bound can be derived in closed-form as follows (see Appendix B for the proof):

$$
P_{\mathrm{out}}^{\mathrm{ub}}=\prod_{l=1}^{L}\left(\sum_{i=1}^{2} F_{\gamma_{U_{i, l}^{\min }}^{\mathrm{m}}}\left(\gamma_{t h}\right)-\prod_{i=1}^{2} F_{\gamma_{U_{i, l}^{\min }}^{\mathrm{lb}}}\left(\gamma_{t h}\right)\right),
$$

where $F_{\gamma_{U_{i, l}^{\mathrm{min}}}^{\mathrm{lb}}}\left(\gamma_{t h}\right)$ is the CDF of $\gamma_{U_{i, l}^{\min }}^{\mathrm{lb}}$ and is defined in (16).

Next, an asymptotic high SNR approximation of the upper bound of the overall outage probability can be derived by using (32) as follows (see Appendix C for the proof):

$$
P_{\mathrm{out}}^{\mathrm{ub}, \infty}=\left(\prod_{l=1}^{L} \Lambda_{l}\right)\left(\frac{\gamma_{t h}}{\bar{\gamma}_{U, R}}\right)^{d_{\mathrm{ub}}}+o\left(\bar{\gamma}_{U, R}^{-\left(d_{\mathrm{ub}}+1\right)}\right),
$$

where $\bar{\gamma}_{1, l}=\bar{\gamma}_{2, l}=\bar{\gamma}_{U, R_{l}}, \bar{\gamma}_{l, 1}=\bar{\gamma}_{l, 2}=\bar{\gamma}_{R_{l}, U}, \bar{\gamma}_{R_{l}, U}=\nu_{l} \bar{\gamma}_{U, R_{l}}$, and $\bar{\gamma}_{U, R_{l}}=C_{l} \bar{\gamma}_{U, R}$. In (33), $d_{u b}$ is an upper bound of the achievable diversity order and can be derived as

$$
d_{\mathrm{ub}}=\sum_{l=1}^{L}\left(\min \left(N_{1}, N_{2}\right)-N_{R_{l}}+1\right) .
$$

\footnotetext{
${ }^{2}$ Our analytical and simulation results reveal that this outage upper bound is asymptotically parallel to the exact outage curves and converges to the exact outage whenever $N_{R_{l}} \rightarrow 1$ for $l \in\{1, \cdots, L\}$ (see Fig. 1) in Section V.
}

Besides, in (33), $\Lambda_{l}$ can be derived as follows [11]:

$$
\Lambda_{l}= \begin{cases}\frac{\eta_{1, l}\left(\frac{N_{1}+N_{2}-2 N_{R_{l}}}{N_{2}-N_{R_{l}}}\right)^{N_{1}-N_{R_{l}}+1}}{\left(N_{1}-N_{R_{l}}+1\right) C_{l} C_{1}-N_{R_{l}}+1}, & N_{1}<N_{2} \\ \frac{\eta_{2, l}\left(\frac{N_{1}+N_{2}-2 N_{R_{l}}}{N_{1}-N_{R_{l}}}\right)^{N_{2}-N_{R_{l}}+1}}{\left(N_{2}-N_{R_{l}}+1\right) C_{l}-N_{2}-N_{R_{l}}+1} & , N_{2}<N_{1} \\ \frac{\left(\eta_{1, l}+\eta_{2, l}\right) 2^{N-N_{R_{l}}+1}}{\left(N-N_{R_{l}}+1\right) C_{l}^{N-N_{R_{l}}+1}}, & N_{1}=N_{2}=N,\end{cases}
$$

Moreover, in (35), $\eta_{i, l}$ for $i \in\{1,2\}$ can be defined as follows:

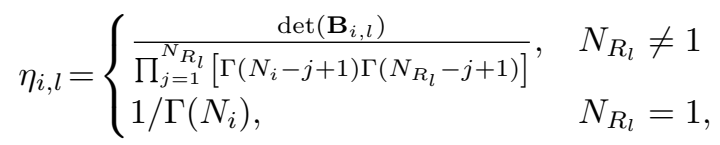

where $\mathbf{B}_{i, l}$ is an $\left(N_{R_{l}}-1\right) \times\left(N_{R_{l}}-1\right)$ matrix with the $(p, q)$ th element given by $\left[\mathbf{B}_{i, l}\right]_{p, q}=\Gamma\left(N_{i}-N_{R_{l}}+p+q+1\right)$ for $i \in$ $\{1,2\}$.

2) Lower bound on the overall outage probability: A lower bound of the overall outage probability can then be defined by using the SNR upper bound in (18) as ${ }^{3}$

$$
P_{\text {out }}^{\mathrm{lb}}=\operatorname{Pr}\left[\max _{l \in\{1, \cdots, L\}}\left(\min \left(\gamma_{U_{1, l}^{\min }}^{\mathrm{ub}}, \gamma_{U_{2, l}^{\min }}^{\mathrm{ub}}\right)\right) \leq \gamma_{t h}\right],
$$

where $\gamma_{U_{i, l}^{\min }}^{\mathrm{ub}}$ is an upper bound for the smallest data substream SNR at $U_{i}^{i}$ received via the $l$ th relay and is defined in (18). Next, the outage upper bound can be derived in closed-form as (see Appendix B for the proof)

$$
P_{\mathrm{out}}^{\mathrm{lb}}=\prod_{l=1}^{L}\left(\sum_{i=1}^{2} F_{\gamma_{U_{i, l}^{\mathrm{min}}}^{\mathrm{ub}}}\left(\gamma_{t h}\right)-\prod_{i=1}^{2} F_{\gamma_{U_{i, l}^{\mathrm{min}}}^{\mathrm{ub}}}\left(\gamma_{t h}\right)\right),
$$

where the CDF of $\gamma_{U_{i, l}^{\min }}^{\mathrm{ub}}$ is defined in (19).

Next, the asymptotic high SNR approximation for the lower bound of the overall outage probability can be derived as follows (see Appendix C for the proof):

$$
P_{\mathrm{out}}^{\mathrm{lb}, \infty}=\left(\prod_{l=1}^{L} \Theta_{l}\right)\left(\frac{\gamma_{t h}}{\bar{\gamma}_{U, R}}\right)^{d_{\mathrm{lb}}}+o\left(\bar{\gamma}_{U, R}^{-\left(d_{\mathrm{lb}}+1\right)}\right),
$$

where $d_{\mathrm{lb}}$ is a lower bound of the achievable diversity order and is given by

$$
d_{\mathrm{lb}}=\sum_{l=1}^{L}\left(\min \left(N_{1}, N_{2}\right)-N_{R_{l}}+1\right) .
$$

Further, in (39), $\Theta_{l}$ is defined as follows:

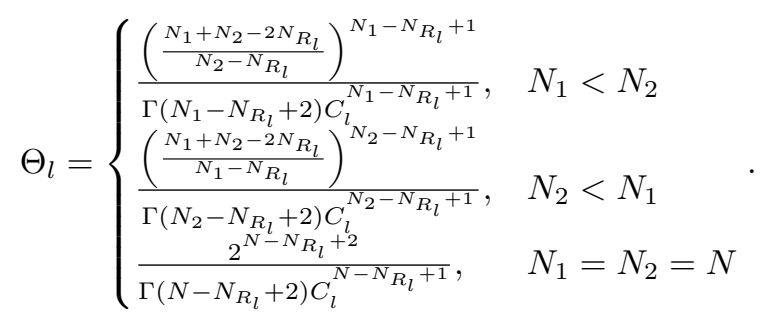

\footnotetext{
${ }^{3}$ Again, our analytical and simulation results clearly show that this outage lower bound is asymptotically parallel to the exact outage curves and converges to the exact outage whenever $N_{R_{l}} \rightarrow 1$ for $l \in\{1, \cdots, L\}$ (see Fig. 1) in Section V.
} 


\section{B. Diversity-multiplexing trade-off of SS-1}

Multiple antennas can be used to increase diversity order or spatial multiplexing gains in wireless systems. The seminal work of Zheng and Tse [10] showed that both types of these gains can be simultaneously obtained for a given multiple-antenna channel subjected to a fundamental trade-off. Thus, the multiplexing gain comes at the expense of sacrificing the diversity gain and vice versa. In this section, we mainly focus on quantifying the diversity-multiplexing trade-off (DMT) of the proposed SS-1. To this end, two DMT expressions are derived by using the lower and upper bound of the asymptotic high SNR approximations of the outage probability, and thereby, the achievable DMT, maximum diversity order, and spatial multiplexing gain are quantified in closed-form.

To begin with, the diversity order and spatial multiplexing gain can be defined as follows [10]:

$$
d=-\lim _{\gamma \rightarrow \infty} \frac{\log \left(P_{\text {out }}(\mathcal{R}, \gamma)\right)}{\log (\gamma)} \quad \text { and } r=\lim _{\gamma \rightarrow \infty} \frac{\mathcal{R}(\gamma)}{\log (\gamma)},
$$

where $P_{\text {out }}(\mathcal{R}, \gamma)$ is the information rate outage probability evaluated at $\gamma$ while keeping the threshold rate at $\mathcal{R}$. Further, $\mathcal{R}(\gamma)$ is the achievable data rate evaluated at the $\gamma$. Next, by using (42), the DMT can be defined as [10]

$$
d(r)=-\lim _{\gamma \rightarrow \infty} \frac{\log \left(P_{\text {out }}(r \log (\gamma))\right)}{\log (\gamma)} .
$$

In the following subsections, the achievable DMT of best relay selection for MIMO TWRNs is quantified by using (43).

1) DMT by using outage lower bound: To begin with, the DMT is quantified by using the asymptotic outage lower bound in (39). In this context, the achievable mutual information can be upper bounded as

$$
\begin{aligned}
\mathcal{I}_{\mathrm{ub}}= & \left(\min _{l \in\{1, \cdots, L\}}\left(N_{R_{l}}\right)\right) \\
& \times \log \left(1+\max _{l \in\{1, \cdots, L\}}\left(\min \left(\gamma_{U_{1, l}^{\min }}^{\mathrm{ub}}, \gamma_{U_{2, l}^{\min }}^{\mathrm{ub}}\right)\right)\right),
\end{aligned}
$$

where $\gamma_{U_{i, l}^{\min }}^{\mathrm{ub}}$ is defined in (18). A lower bound of the information outage probability can be then defined by using (44) as follows:

$$
P_{\text {out }}^{\mathrm{lb}}=\operatorname{Pr}\left(\mathcal{I}_{\mathrm{ub}} \leq \mathcal{R}_{t h}\right),
$$

where $\mathcal{R}_{t h}$ is the pre-set threshold rate of the overall system and is defined as $\mathcal{R}_{t h}=r \log \left(1+\bar{\gamma}_{U, R}\right)$ [31]. By substituting (44) into (45), the outage lower bound can be written in an alternative form as follows:

$P_{\text {out }}^{\mathrm{lb}}=\operatorname{Pr}\left(\max _{l \in\{1, \cdots, L\}}\left(\min \left(\gamma_{U_{1, l}^{\min }}^{\mathrm{ub}}, \gamma_{U_{2, l}^{\min }}^{\mathrm{ub}}\right)\right) \leq\left(\bar{\gamma}_{U, R}\right)^{\frac{r}{N_{R}^{\min }}}-1\right)$,

where $N_{R}^{\min }=\min _{l \in\{1, \cdots, L\}} N_{R_{l}}$. By using (39), the outage lower bound in (46) can be asymptotically approximated when $\bar{\gamma}_{U, R} \rightarrow$ $\infty$ as follows:

$$
P_{\text {out }}^{\mathrm{lb}, \infty} \approx \bar{\gamma}_{U, R}^{-\sum_{l=1}^{L}\left(\min \left(N_{1}, N_{2}\right)-N_{R_{l}}+1\right)\left(1-\frac{r}{N_{R}^{\min }}\right) .} .
$$

The lower bound of the achievable DMT can then be derived by using its definition in [31] as

$$
d^{\mathrm{lb}}(r)=\left(\sum_{l=1}^{L}\left(\min \left(N_{1}, N_{2}\right)-N_{R_{l}}+1\right)\right)\left(1-\frac{r}{N_{R}^{\min }}\right) .
$$

2) DMT by using outage upper bound: A lower bound of the achievable mutual information can be written as

$$
\mathcal{I}_{\mathrm{lb}}=N_{R}^{\min } \log \left(1+\max _{l \in\{1, \cdots, L\}}\left(\min \left(\gamma_{U_{1, l}^{\min }}^{\mathrm{lb}} \gamma_{U_{2, l}^{\min }}^{\mathrm{lb}}\right)\right)\right)
$$

where $\gamma_{U_{i, l}^{\min }}^{\mathrm{lb}}$ is defined in (15). Then, the corresponding information outage probability upper bound can be defined by using (49) as

$$
P_{\text {out }}^{\mathrm{lb}}=\operatorname{Pr}\left(\mathcal{I}_{\mathrm{lb}} \leq \mathcal{R}_{t h}\right),
$$

By substituting (49) into (50), the upper bound of the outage probability can be then written as

$P_{\text {out }}^{\mathrm{ub}}=\operatorname{Pr}\left(\max _{l \in\{1, \cdots, L\}}\left(\min \left(\gamma_{U_{1, l}^{\min }}^{\mathrm{lb}}, \gamma_{U_{2, l}^{\min }}^{\mathrm{lb}}\right)\right) \leq\left(\bar{\gamma}_{U, R}\right)^{\frac{r}{N_{R}^{\min }}}-1\right)$

An asymptotic high SNR approximation of the outage upper bound in (51) can be derived as

$$
P_{\mathrm{out}}^{\mathrm{ub}, \infty} \approx \bar{\gamma}_{U, R}^{-\sum_{l=1}^{L}\left(\min \left(N_{1}, N_{2}\right)-N_{R_{l}}+1\right)\left(1-\frac{r}{N_{R}^{\min }}\right) .} .
$$

The corresponding upper bound of the achievable DMT can then be derived as

$$
d^{\mathrm{ub}}(r)=\left(\sum_{l=1}^{L}\left(\min \left(N_{1}, N_{2}\right)-N_{R_{l}}+1\right)\right)\left(1-\frac{r}{N_{R}^{\min }}\right) .
$$

Remark IV.1: Note that the DMT derived by using the lower and upper bounds of the overall outage probability given in (48) and (53), respectively, are the same. Thus, it can be concluded that the achievable diversity order is given by

$d(r)=\left(\sum_{l=1}^{L}\left(\min \left(N_{1}, N_{2}\right)-N_{R_{l}}+1\right)\right)\left(1-\frac{r}{\min _{l \in\{1, \cdots, L\}}\left(N_{R_{l}}\right)}\right)$

This DMT (54) provides several important insights. The maximum achievable diversity order and the spatial multiplexing gain can be derived as

$$
\begin{aligned}
d_{\max } & =\sum_{l=1}^{L}\left(\min \left(N_{1}, N_{2}\right)-N_{R_{l}}+1\right) \\
& =L\left(\min \left(N_{1}, N_{2}\right)+1\right)-\sum_{l=1}^{L} N_{R_{l}} \text { and } \\
r_{\max } & =\min _{l \in\{1, \cdots, L\}} N_{R_{l}} .
\end{aligned}
$$

Thus, the spatial multiplexing gain of SS-1 is solely governed by the number of antennas at the relay which has the smallest antenna array $\left(N_{R}^{\min }\right)$. However, whenever $N_{R}^{\min }$ is increased for a given $N_{1}$ and $N_{2}$ in order to increase the multiplexing gain, the achievable diversity order decreases. This decrease in diversity order is more prominent when the MIMO TWRN operates without the best relay selection (i.e., $L=1$ ). The best relay selection based on SS-1 alone does not increase the achievable spatial multiplexing gain as it is independent of the number of available relays $(L)$. Nevertheless, the multiplexing gain can indeed be improved by maximizing minimum number of antennas at any relay. Counter-intuitively, the best relay selection based on SS-1 improves the diversity order by a multiplicative 


$$
\begin{gathered}
\overline{\mathcal{R}}_{L^{*}} \approx \\
\frac{N_{R}^{\max }}{\ln (2)} \sum_{m=0}^{N-N_{R}^{\max }} \sum_{n=0}^{N-N_{R}^{\max }} \frac{1}{2^{m+n} m ! n !}\left(\mathbb{J}\left(m+n, 2 \zeta_{L^{*}}, \alpha_{L^{*}}+\beta_{L^{*}}\right)-\mathbb{J}\left(m+n, 2 \zeta_{L^{*}}, \beta_{L^{*}}\right)\right. \\
\left.-(m+n)\left(\mathbb{J}\left(m+n-1,2 \zeta_{L^{*}}, \alpha_{L^{*}}+\beta_{L^{*}}\right)-\mathbb{J}\left(m+n-1,2 \zeta_{L^{*}}, \beta_{L^{*}}\right)\right)\right) .
\end{gathered}
$$

$\mathbb{J}(x, y, z)=\int_{0}^{\infty} \lambda^{x} \exp (-\lambda) \ln (y+z \lambda) \mathrm{d} \lambda=\Gamma(x+1)\left(\ln (y)+\sum_{p=0}^{x} \frac{1}{\Gamma(x-p+1)}\left(\left(\frac{-y}{z}\right)^{x-p} \exp \left(\frac{y}{z}\right) E_{1}\left(\frac{y}{z}\right)+\sum_{q=1}^{x-p} \Gamma(q)\left(\frac{-y}{z}\right)^{x-p-q}\right)\right)$.

$\overline{\mathcal{R}}_{L^{*}} \approx \frac{N_{R}}{\ln (2)} \sum_{l=1}^{L} \sum_{k=1}^{2 l\left(N-N_{R}\right)}(-1)^{l}\left(\begin{array}{l}l \\ L\end{array}\right) \frac{\beta_{k, 2 l, N-N_{R}+1}}{(2 l)^{k}}((k \mathbb{J}(k-1,2 l \zeta, \alpha+\beta)+\mathbb{J}(k, 2 l \zeta, \beta)-k \mathbb{J}(k-1,2 l \zeta, \beta)-\mathbb{J}(k, 2 l \zeta, \alpha+\beta)))$.

factor of $L$ whenever $N_{R_{l}}=N_{R}$ for $l \in\{1, \cdots, L\}$, and thereby, compensates the diversity order degradation whenever $N_{R}$ is increased in an attempt to improve the multiplexing gain.

Interestingly, the second equality of $d_{\max }$ in (55) reveals that whenever the sum of the relay antennas is fixed, the maximum diversity order is always a constant. In this context, the multiplexing gain can readily be improved by equally distributing antennas among the available set of relays.

\section{Sum rate analysis of SS-2}

In this subsection, the sum rate of the best relay selection based on maximizing the sum rate (SS-2) is investigated. Again, the exact derivation of the ergodic sum rate of SS-2 appears mathematically intractable due to statistical dependence of data substream SNRs belonging to a given user node (see remark II.1). Thus, in this context, an approximation of the ergodic sum rate can be derived for obtaining useful insights. To begin with, the sum of the rates of data substreams of $U_{i}$ (24) can be approximated by its lower bound as

$$
\mathcal{R}_{U_{i, l}}=\frac{1}{2} \sum_{k=1}^{N_{R_{l}}} \log \left(1+\gamma_{U_{i, l}^{(k)}}\right) \approx \frac{N_{R_{l}}}{2} \log \left(1+\gamma_{U_{i, l}^{\min }}\right)
$$

where $\gamma_{U_{i, l}^{\min }}$ is defined in (18), $i \in\{1,2\}$ and $l \in\{1, \cdots, L\}$. By first substituting (56) into (23), and then by performing several mathematical formulations, an approximation for the sum rate for the best relay selection based on SS-2 can then be written as follows ${ }^{4}$ :

$$
\mathcal{R}_{L^{*}} \approx\left(\max _{l \in\{1, \cdots, L\}}\left(N_{R_{l}}\right)\right) \log \left(1+\min \left(\gamma_{U_{1, L^{*}}^{\min }}, \gamma_{U_{2, L^{*}}^{\min }}\right)\right) .
$$

By using (18), $\gamma_{U_{i, L^{*}}^{\min }}$ for $i \in\{1,2\}$ in (57) can be approximated as

$$
\gamma_{U_{i, L^{*}}} \approx \frac{\alpha_{i, L^{*}}}{\beta_{i, L^{*}}+\zeta_{i, L^{*}}\left[\left(\mathbf{F}_{L^{*}, i}^{H} \mathbf{F}_{L^{*}, i}\right)^{-1}\right]_{k, k}}
$$

\footnotetext{
${ }^{4}$ It is worth noting that the sum rate approximation in (57) provides very close results as shown by our extensive Monte-Carlo simulations in Fig. 4 presented in Section V.
}

where $k \in\left\{1, \cdots, N_{R}^{\max }\right\}$. Further, $\alpha_{i, L^{*}}, \beta_{i, L^{*}}$, and $\zeta_{i, L^{*}}$ are defined as follows:

$$
\begin{aligned}
& \alpha_{i, L^{*}}=\left(N_{i^{\prime}}-N_{R}^{\max }\right) \bar{\gamma}_{i^{\prime}, L^{*}} \bar{\gamma}_{L^{*}, i}, \\
& \beta_{i, L^{*}}=N_{R}^{\max } \bar{\gamma}_{L^{*}, i}, \\
& \zeta_{i, L^{*}}=\left(N_{i^{\prime}}-N_{R}^{\max }\right) \bar{\gamma}_{i^{\prime}, L^{*}}+\left(N_{i}-N_{R}^{\max }\right) \bar{\gamma}_{i, L^{*}}+N_{R}^{\max } .
\end{aligned}
$$

Here, in (58b), (58c), and (58d), $N_{R}^{\max }=\max _{l \in\{1, \cdots, L\}} N_{R_{l}}, i \in$ $\{1,2\}, i^{\prime} \in\{1,2\}$, and $i \neq i^{\prime}$. The average sum rate approximation can next be derived by taking the expectation of $\mathcal{R}_{L^{*}}$ in (57) over the corresponding SNR, $\gamma_{\mathrm{eq}}=\min \left(\gamma_{U_{1, L^{*}}^{\min }}, \gamma_{U_{2, L^{*}}^{\min }}\right)$, as shown in (59) on the top of this page (see Appendix D for the proof). Here in (59), the function $\mathbb{J}(x, y, z)$ is defined in (60).

Remark IV.2: The close-form approximation of the ergodic sum rate presented in (59) is valid for different number of relay antennas $\left(N_{R_{l}}\right.$ for $\left.l \in\{1, \cdots, L\}\right)$. Nevertheless, our numerical results reveal that this approximation slightly weakens when all available relays are equipped with the same number of antennas $\left(N_{R_{l}}=N_{R}\right.$ for $\left.l \in\{1, \cdots, L\}\right)$. Thus, for this case, a tight ergodic sum rate approximation can next be derived explicitly as given in (61) (see Appendix E for the proof). Here, in (61), $\alpha=\alpha_{i, l}, \zeta=\zeta_{i, l}$, and $\beta=\beta_{i, l}$ for all $i \in\{1,2\}$ and $l \in\{1, \cdots, L\}$. The multinomial coefficient $\beta_{k, 2 l, N-N_{R}+1}$ in (61) can be written as

$$
\beta_{k, 2 l, N-N_{R}+1}=\sum_{i=k-N+N_{R}}^{k} \frac{\beta_{i, 2 l-1, N-N_{R}+1}}{(k-i) !} I_{\left[0,(2 l-1)\left(N-N_{R}\right)\right]}(i) .
$$

In (62), $\beta_{0,0, N-N_{R}+1}=\beta_{0,2 l, N-N_{R}+1}=1, \beta_{k, 1, N-N_{R}+1}=$ $1 / k !, \beta_{1,2 l, N-N_{R}+1}=2 l, I_{[a, c]}(b)=1$ for $a \leq b \leq c$, and $I_{[a, c]}(b)=0$ otherwise.

\section{Diversity-multiplexing trade-off of SS-2}

By following similar steps to those in Section IV-B, the DMT of SS-2 can be derived as follows ${ }^{5}$ :

$$
\begin{array}{r}
d(r)=\left(\sum_{l=1}^{L^{\prime}}\left(\min \left(N_{1}, N_{2}\right)-\max _{l \in\{1, \cdots, L\}}\left(N_{R_{l}}\right)+1\right)\right) \\
\times\left(1-\frac{r}{\max _{l \in\{1, \cdots, L\}}\left(N_{R_{l}}\right)}\right),
\end{array}
$$

\footnotetext{
${ }^{5}$ The proof of the DMT of SS-2 (63) is omitted due to the page restrictions and its repetitive nature to the proof of (54).
} 


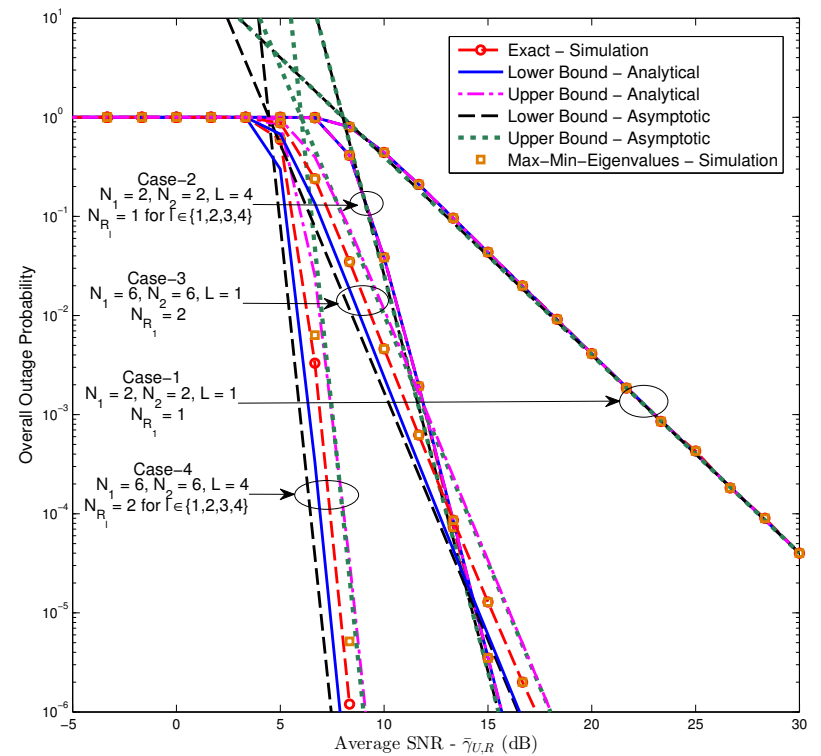

Fig. 1. The overall outage probability of the best relay selection based on SS-1 for MIMO AF TWRNs with equal number of antennas at relays. The average transmit SNRs $\bar{\gamma}_{i, l}$ and $\bar{\gamma}_{l, i}$ for $i \in\{1,2\}$ and $l \in\{1, \cdots, L\}$ are assumed equal and denoted as $\bar{\gamma}_{U, R}$.

where $L^{\prime}$ is the number of relays having $N_{R}^{\max }=$ $\max _{l \in\{1, \cdots, L\}}\left(N_{R_{l}}\right)$ number of antennas. Interestingly, by comparing (54) and (63), it can be noted that the DMT of SS-2 becomes identical to that of SS-1 whenever all available relays are equipped with the same number of antennas (i.e., $N_{R_{l}}=N_{R}$ for $i \in\{1, \cdots, L\})$.

\section{NumericAl Results}

In this section, numerical and simulation results are presented to investigate the performance of our proposed selection strategies. To this end, the overall outage probability, average sum rate, and diversity-multiplexing curves are plotted. These plots are generated by using both analytical expressions and MonteCarlo simulations. As an aside, they justify the validity of our analysis.

\section{A. Overall outage probability of SS-1}

In Fig. 1, the performance of the best relay selection based on maximizing the SNR of the worst data substream at the weakest user node or equivalently minimizing the outage probability (SS1 ) is plotted for four specific system set-ups ${ }^{6}$ (see case- 1 to case4 in Fig. 1). To this end, the upper and lower outage bounds are plotted by using (32) and (38), respectively. The outage probability curves for $L=1$ (i.e., no relay selection) are plotted for comparison purposes. The outage curves of case-1/case- 2 and case-3/case-4 reveal the significant performance gains of best relay selection. For example, at an outage probability of $10^{-5}$, selecting the best out of four relays (case-4) provides almost $7.5 \mathrm{~dB}$ SNR gain over no relay selection (case-3). Further, our asymptotic outage curves reveal that selecting the best out of

\footnotetext{
${ }^{6}$ These outage curves are plotted for TWRNs with relays having equal number of relay antennas for a given case. The outage curves for TWRNs with relays having different number of antennas are plotted separately in Fig. 2 to avoid lack of clarity due to cluttering of the curves.
}



Fig. 2. The overall outage probability of best relay selection (SS-1) for MIMO AF TWRNs with different number of antennas at relays. The average transmit SNRs $\bar{\gamma}_{i, l}$ and $\bar{\gamma}_{l, i}$ for $i \in\{1,2\}$ and $l \in\{1, \cdots, L\}$ are assumed equal and denoted as $\bar{\gamma}_{U, R}$

four relays (case -2) provides four-times the diversity order that is achieved by no relay selection (case-1). Besides, Fig. 1 clearly reveals that our upper and lower outage bounds approach to the simulated curves for single-antenna relay TWRNs. Further, the outage curves obtained by maximizing the minimum of the eigenvalues of the Wishart matrices closely follow the simulated outage curves obtained via (20), and this observation justifies the validity of (22).

Fig. 2, the overall outage probability of SS-1 is plotted for three relays equipped with different number of antennas. The high SNR outage asymptotics are plotted by using (33) and (39) to investigate the achievable diversity order by three specific system configurations (see case-1, case-2, and case-3 in Fig. 2.). The outage curves are plotted by using Monte-Carlo simulations for diversity order comparison purposes. Fig. 2 shows that the achievable diversity order of case- 1 , case- 2 , and case- 3 are eleven, nine, and seven respectively. These diversity orders match with those of (55), validating our diversity order analysis. Moreover, for a fixed number of antennas at the two user nodes and a fixed number of relays, the system set-up having the least number of aggregate relay antennas provides the highest diversity order, whereas, the system set-up having the highest number of aggregate relay antennas provides the least diversity order. Intuitively, the achievable overall diversity order by the best relay selection based on SS-1 is given by the aggregate of the diversity orders of each end-to-end relayed-channel.

\section{B. Sum rate of SS-2}

In Fig. 3, the sum rate of the best relay selection based on maximizing the sum rate (SS-2) is investigated when the number of antennas at each relay is fixed to three. Six different cases are plotted to obtain insights about the achievable sum rate performance of SS-2. Monte-Carlo simulations and the sum rate approximations from (61) are plotted. Fig. 3 clearly reveals that 




Fig. 3. The achievable average sum rate of the best relay selection based on SS2 for MIMO AF TWRNs with equal number of antennas at relays. The average transmit SNRs $\bar{\gamma}_{i, l}$ and $\bar{\gamma}_{l, i}$ for $i \in\{1,2\}$ and $l \in\{1, \cdots, L\}$ are assumed equal and denoted as $\bar{\gamma}_{U, R}$.

the sum rate heavily depends on (i) the relay antenna count, (ii) user node antenna count, and (iii) number of relays. For example, at an SNR of $15 \mathrm{~dB}$, three relays with user nodes each with four antennas (case-2/case-4) achieves a sum rate increase of four bits/channel-use/Hz by upgrading the single-antenna relays (case-2) to triple-antenna relays (case-4). Moreover, at an SNR of $10 \mathrm{~dB}$, for the triple-relay/triple-antenna-relay TWRN, a sum rate gain of 3 bits/channel-use/Hz can be achieved by increasing the number of user antennas from four (case-4) to six (case6). Besides, the triple-relay TWRN with quadruple-antenna users (case-4) provides about a sum rate gain of one bits/channeluse/Hz over the single-relay counterpart (case-3). Fig. 3 shows that the sum rate curves obtained via relay selection based on maximizing the minimum determinant of the Wishart matrices (29) coincides with the simulated sum rate curves. Further, our analytical sum rate approximation is significantly tight to the simulated sum rate curves.

In Fig. 3, we saw how the total number of antennas and relays tends to increase the sum rate. In Fig. 4, we try to isolate the effect of the number of relay antennas. For this purpose, we fix the number of relays to four and plot the sum rate performance with different numbers of relay antennas. Fig. 4 clearly shows that the sum rate is heavily dependent on the number of relay antennas. By comparing sum rate curves of case-1, case-2, case-3, and case4 , we conclude that the achievable sum rate, and hence the spatial multiplexing gain is solely governed by the relay with the largest antenna array. For example, at an SNR of $20 \mathrm{~dB}$, case- 4 provides sum rate gains of $12.5,7$, and 2.5 bits/channel-use/Hz over case-1, case- 2 , and case- 3 , respectively. Intuitively, these sum rate gains are a direct consequence of having a quadruple, triple, and dualantenna relay as the relay with the largest antenna array in case- 4 , case- 3 , and case- 2 , respectively. Further, TWRNs of case- 4 and case- 5 have the same set of relays, however, the TWRNs of the former and latter cases are equipped with sextuple-antenna and octuple-antenna user nodes, respectively. Thus, case-5 provides a

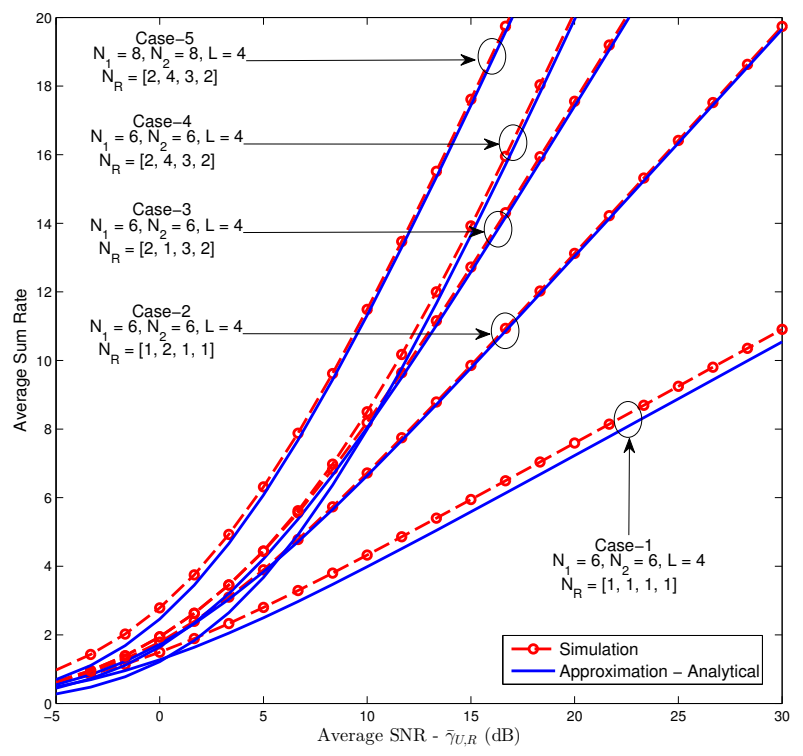

Fig. 4. The achievable average sum rate of the best relay selection based on SS-2 for MIMO AF TWRNs with different number of antennas at relays. The average transmit SNRs $\bar{\gamma}_{i, l}$ and $\bar{\gamma}_{l, i}$ for $i \in\{1,2\}$ and $l \in\{1, \cdots, L\}$ are assumed equal and denoted as $\bar{\gamma}_{U, R}$.

sum rate gain of 3.5 bits/channel-use/Hz over case- 4 , nevertheless, the spatial multiplexing gains of both these cases are the same as the largest relay-antenna array is four for both the cases. Again, the sum rate approximations are plotted by using our analysis (59) and they are fairly tight to the Monte-Carlo plots ${ }^{7}$.

\section{Diversity-multiplexing trade-off}

In Fig. 5a, the achievable diversity-multiplexing trade-off (DMT) of best relay selection strategies for MIMO TWRNs with relays having same number of antennas is investigated. Thus, DMT curves in Fig. 5a are valid for both SS-1 and SS-2 ${ }^{8}$. Here, two sets of DMT curves are plotted by changing the number of relays and size of the relay antenna arrays. Fig. 5a clearly reveals that the diversity order increases whenever the number of available relays $(L)$ is increased, however, the achievable spatial multiplexing gain does not increase with $L$. Counter-intuitively, the achievable diversity order decreases whenever the number of antennas at the relay $\left(N_{R}\right)$ is increased; compensating this trend is the increase of the multiplexing gain. These two observations clearly reveal that the spatial multiplexing gain is solely governed by $N_{R}$. Whenever $N_{R}$ is increased, much of the available degrees of freedom boosts the multiplexing gain, and consequently, the achievable diversity order decreases. Nevertheless, the best relay selection significantly alleviates this inherent reduction of diversity order by increasing the degrees of freedom available for boosting the overall diversity order. Thus, there is a fundamental DMT associated with TWRNs with multiple-data streams, and the best relay selection indeed improves the achievable DMT.

\footnotetext{
${ }^{7}$ The analytical sum rate curve corresponding to case-1 slightly deviates away from the exact sum rate curve. Thus, this observation clearly justifies remark IV-2 and hence the derivation of a tighter sum rate approximation (61) for the TWRNs with relays having the same number of antennas.

${ }^{8}$ Whenever all available relays are equipped with the equal number of antennas, the DMT of SS-1 (43) and DMT of SS-2 (63) become identical.
} 


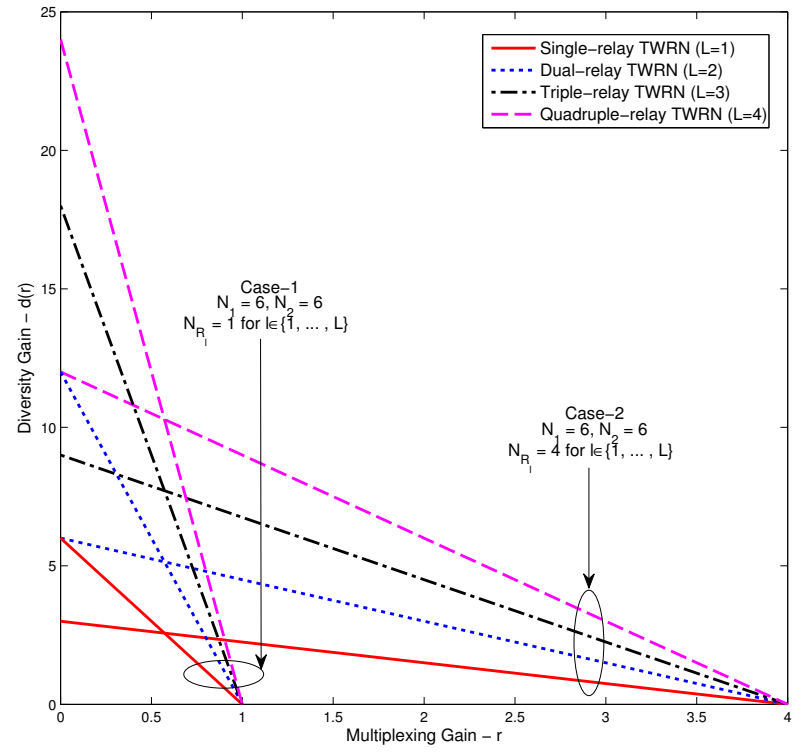

(a) DMT - Relays have same number of antennas

Fig. 5. Diversity-multiplexing trade-off (DMT) comparison.

In Fig. 5b, the achievable DMTs of both SS-1 and SS-2 are studied for MIMO TWRNs whenever the available relays are equipped with different number of antennas. DMT curves of case- 1 and case- 2 clearly reveal that the maximum achievable diversity order of SS-1 is always higher than that of SS-2, whereas SS-2 provides the highest multiplexing gain. This behaviour is not surprising as relay selection based on SS-1 is designed to maximize the effective end-to-end SNR and thereby to maximize the overall diversity order, while the relay selection based on SS-2 is designed to maximize the sum rate.

\section{Outage probability and sum rate comparison between SS-1 and $S S-2$}

In Fig. 6, the overall outage probability and sum rate performances of our proposed relay selection strategies are compared. To this end, in Fig. 6a and Fig. 6b, the sum rate and outage probability, respectively, are plotted for TWRNs with best relay selection based on SS-1 and SS-2 by considering four cases of system configurations. Fig. 6a clearly shows that the sum rate performance of SS-2 is significantly better than that of SS-1. This observation is not surprising as SS-2 is explicitly designed to maximize the sum rate. However, Fig. $6 \mathrm{~b}$ reveals that the outage probability performance of SS-1 is considerably better than that of SS-2. Again, this behaviour is not surprising, as well, because SS-1 is designed to maximize the effective SNR and thereby minimizing the overall outage probability. Counterintuitively, case-1 provides the worst sum rate performance (see Fig. 6a), as well, the best outage probability performance (see Fig. 6b) irrespective of the relay selection strategy (i.e., SS-1 or SS-2). Similarly, case- 3 provides the best sum rate performance and worst outage probability performance. The aforementioned observations are due to direct consequences of the achievable diversity-multiplexing trade-off, and notably, larger relay antenna



(b) DMT - Relays have different number of antennas

arrays indeed increases the multiplexing gain, while reducing the overall diversity order.

\section{CONCLUSION}

In this paper, two novel relay selection strategies were proposed and analyzed for MIMO AF TWRNs with spatial multiplexing. The first strategy maximizes the SNR of the worst data subchannel of the weakest user node, and thereby, minimizing the overall outage probability of the two user nodes. Notably, first strategy is equivalent to selecting the relay which maximizes the minimum of the eigenvalues of the corresponding Wishart matrices. The second strategy maximizes the achievable sum rate by selecting the relay which maximizes the minimum of the determinant of the Wishart matrices. The performance of these strategies was studied by deriving the lower/upper bounds of the overall outage probability and average sum rate approximations in closed-form. Further, achievable diversity order and spatial multiplexing gain were characterized by deriving the fundamental diversity-multiplexing trade. Notably, this trade-off shows that whenever the sum of relay antennas is fixed, then the achievable diversity order always becomes a constant, and hence, the overall multiplexing gain can be improved by equally distributing the antennas among the available set of relays. Several numerical results were presented to compare the performance gains of the proposed relay selection strategies and to validate our analysis. Our results reveal that the proposed relay selection strategies provide substantial improvements in outage probability, diversity order, and the average sum rate. This study thus confirms the potential use of MIMO AF TWRNs in the context of emerging, next generation wireless networks. While this paper is limited to $\mathrm{ZF}$, for future research, various precoding and detection schemes such as maximal ratio transmission, maximal ratio reception and eigen-beamforming maybe investigated. 


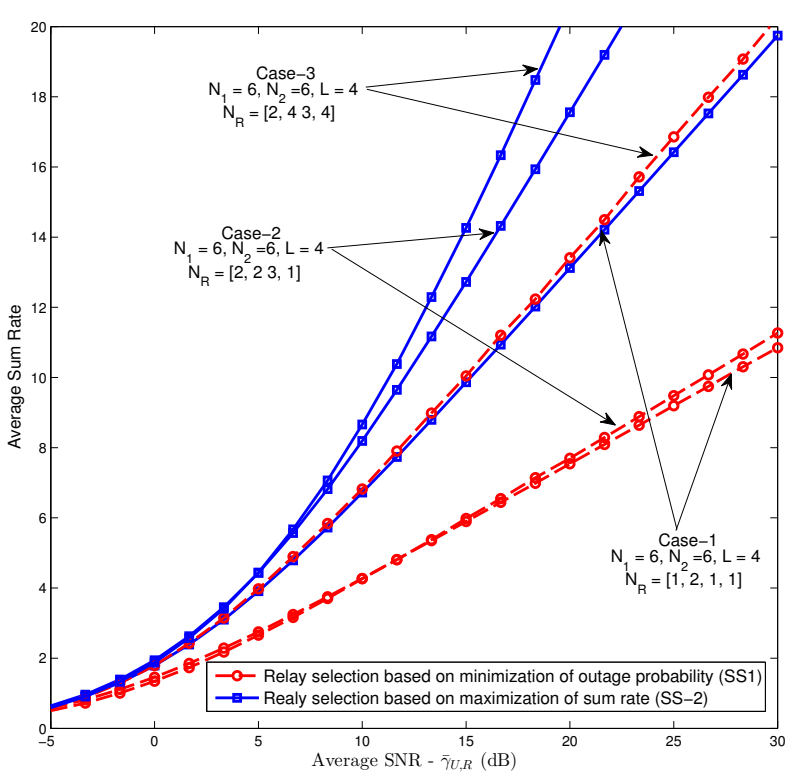

(a) Sum rate comparison

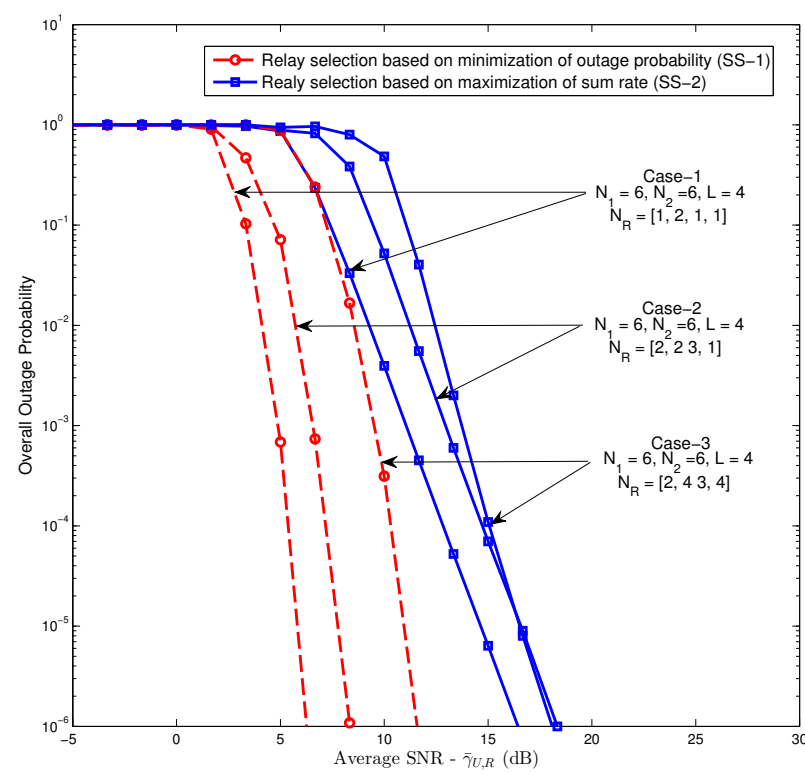

(b) Outage probability comparison

Fig. 6. Performance comparison of two relay selection strategies for MIMO TWRNs with spatial multiplexing. The average transmit SNRs $\bar{\gamma}_{i, l}$ and $\bar{\gamma}_{l, i}$ for $i \in\{1,2\}$ and $l \in\{1, \cdots, L\}$ are assumed equal and denoted as $\bar{\gamma}_{U, R}$.

\section{APPENDIX A}

PROOF OF THE CDFS OF $\gamma_{U_{i, l}^{\min }}^{\mathrm{ub}}$ AND $\gamma_{U_{i, l}^{\min }}^{\mathrm{lb}}$

In this appendix, the proofs of the CDFs of $\gamma_{U_{i, l}^{\min }}^{\mathrm{ub}}$ and $\gamma_{U_{i, l}^{\min }}^{\mathrm{lb}}$ are sketched. To this end, the corresponding SNR can be re-written in an alternative form as follows:

$$
\gamma_{U_{i, l}^{\min }}^{\mathrm{xb}}=\frac{\alpha_{i, l} X_{i, l}}{\beta_{i, l} X_{i, l}+\zeta_{i, l}},
$$

where $X_{i, l}=\left(\left[\left(\mathbf{F}_{l, i}^{H} \mathbf{F}_{l, i}\right)^{-1}\right]_{k, k}\right)^{-1}$ for $\gamma_{U_{i, l}^{\min }}^{\mathrm{ub}}$ and $X_{i, l}=$ $\lambda_{\min }\left\{\mathbf{F}_{l, i}^{H} \mathbf{F}_{l, i}\right\}$ for $\gamma_{U_{i, l}^{\min }}^{\mathrm{lb}}$. The CDF of $\gamma_{U_{i, l}^{\min }}^{\mathrm{xb}}$ can then be written as [11]

$$
F_{\gamma_{U_{i, l}^{\mathrm{mb}}}^{\mathrm{min}}}(z)=\operatorname{Pr}\left(X_{i, l} \leq \frac{\zeta_{i, l} z}{\alpha_{i, l}-\beta_{i, l} z}\right)=F_{X_{i, l}}\left(\frac{\zeta_{i, l} z}{\alpha_{i, l}-\beta_{i, l} z}\right),
$$

where $z<\frac{\alpha_{i, l}}{\beta_{i, l}}$. Next, $F_{\gamma_{U_{i, l}^{\min }}^{\mathrm{ub}}}(z)$ and $F_{\gamma_{U_{i, l}^{\min }}^{\mathrm{lb}}}(z)$ can be derived by evaluating the CDFs of the $k$ th diagonal element of the inverse Wishart matrix [28] and the smallest eigenvalue of the Wishart matrix [27] at $\zeta_{i, l} z /\left(\alpha_{i, l}-\beta_{i, l} z\right)$.

\section{APPENDIX B}

\section{PROOF OF THE OVERALL OUTAGE PROBABILITY BOUNDS}

In this appendix, the proof of the overall outage probability bounds is sketched. To begin with, the definition of the overall outage upper bound is re-written as

$$
P_{\mathrm{out}}^{\mathrm{ub}}=\operatorname{Pr}\left[\gamma_{\mathrm{eq}}^{\mathrm{lb}} \leq \gamma_{t h}\right]=F_{\gamma_{\mathrm{eq}}^{\mathrm{lb}}}\left(\gamma_{t h}\right)
$$

where $\gamma_{\text {eq }}^{\mathrm{lb}}$ is the equivalent SNR and is defined as

$$
\gamma_{\mathrm{eq}}^{\mathrm{lb}}=\max _{l \in\{1, \cdots, L\}}\left(\gamma_{l}^{\mathrm{lb}}\right)
$$

where $\gamma_{l}^{\mathrm{lb}}=\min \left(\gamma_{U_{1, l}^{\min }}^{\mathrm{lb}}, \gamma_{U_{2, l}^{\min }}^{\mathrm{lb}}\right)$, for $l \in\{1, \cdots, L\}$. Next, the CDF of $\gamma_{l}^{\text {lb }}$ can be derived as [32]

$$
F_{\gamma_{l}^{\mathrm{lb}}}(x)=1-\left(1-F_{\gamma_{U_{1, l}^{\min }}^{\mathrm{mb}}}(x)\right)\left(1-F_{\gamma_{U_{2, l}^{\min }}^{\mathrm{lb}}}(x)\right) .
$$

Then, the CDF of $\gamma_{\mathrm{eq}}^{\mathrm{lb}}$ can be derived as follows [32]:

$$
F_{\gamma_{\mathrm{eq}}^{\mathrm{lb}}}(x)=\operatorname{Pr}\left(\gamma_{1}^{\mathrm{lb}} \leq x, \cdots, \gamma_{L}^{\mathrm{lb}} \leq x\right)=\prod_{l=1}^{L}\left(F_{\gamma_{l}^{\mathrm{lb}}}(x)\right) .
$$

Finally, by substituting (68) into (67), the upper bounds of the overall outage probability can be derived as shown in (32). The derivation of the lower bound of the overall outage probability follows the same techniques in (67), (68), and (69), and hence its proof is omitted.

\section{APPENDIX C}

\section{PROOF OF THE ASYMPTOTIC HIGH SNR OUTAGE APPROXIMATIONS}

In this appendix, the proof of the asymptotic outage lower bound is first sketched, and thereby, the asymptotic outage upper bound is deduced: To begin with, the PDF of $\gamma_{U_{i, l}^{\min }}^{\mathrm{ub}}$ for $i \in\{1,2\}$ is derived by differentiating (19) by using the Leibniz integral rule as follows:

$$
\begin{aligned}
f_{\gamma_{U_{i, l}^{\min }}^{\mathrm{ub}}}(x) & =\frac{d}{\mathrm{~d} x}\left[\frac{\zeta_{i, l} x}{\alpha_{i, l}-\beta_{i, l} x}\right]\left(\frac{\zeta_{i, l} x}{\alpha_{i, l}-\beta_{i, l} x}\right)^{N_{i}-N_{R_{l}}} \frac{\mathrm{e}^{-\frac{\zeta_{i, l} x}{\alpha_{i, l}-\beta_{i, l} x}}}{\Gamma\left(N_{i}-N_{R_{l}}+1\right)} \\
& =\frac{\beta_{i, l}\left(\zeta_{i, l}\right)^{N_{i}-N_{R_{l}}+1} x^{N_{i}-N_{R_{l}}} \mathrm{e}^{-\frac{\zeta_{i, l} x}{\alpha_{i, l}-\beta_{i, l} x}}}{\Gamma\left(N_{i}-N_{R_{l}}+1\right)\left(\alpha_{i, l}-\beta_{i, l} x\right)^{N_{i}-N_{R_{l}}+2}}
\end{aligned}
$$

where $0 \leq x<\frac{\alpha_{i, l}}{\beta_{i, l}}$. By substituting $\zeta_{i, l}, \alpha_{i, l}, \beta_{i, l}$, defined in (15) into (70), and then by taking the Taylor series expansion around 


$$
\begin{aligned}
& F_{\gamma_{l}^{\text {ub }}}^{x \rightarrow 0}(x)=\frac{\phi_{1}^{N_{1}-N_{R_{l}}+1}}{\Gamma\left(N_{1}-N_{R_{l}}+2\right) C_{l}^{N_{1}-N_{R_{l}}+1}}\left(\frac{x}{\bar{\gamma}_{U, R}}\right)^{N_{1}-N_{R_{l}}+1}+\frac{\phi_{2}^{N_{1}-N_{R_{l}}+1}}{\Gamma\left(N_{2}-N_{R_{l}}+2\right) C_{l}^{N_{2}-N_{R_{l}}+1}}\left(\frac{x}{\bar{\gamma}_{U, R}}\right)^{N_{2}-N_{R_{l}}+1} \\
& +\frac{\phi_{1}^{N_{1}-N_{R_{l}}+1} \phi_{2}^{N_{2}-N_{R_{l}}+1}}{\Gamma\left(N_{1}-N_{R_{l}}+2\right) \Gamma\left(N_{2}-N_{R_{l}}+2\right) C_{l}^{\left(N_{1}-N_{R_{l}}+1\right)\left(N_{2}-N_{R_{l}}+1\right)}}\left(\frac{x}{\bar{\gamma}_{U, R}}\right)^{\left(N_{1}-N_{R_{l}}+1\right)\left(N_{2}-N_{R_{l}}+1\right)}+o\left(x^{\left(N_{1}-N_{R_{l}}+1\right)\left(N_{2}-N_{R_{l}}+1\right)}\right)
\end{aligned}
$$

$x=0$, the first order expansion ${ }^{9}$ of $f_{\gamma_{S_{i}, \text { min }}^{\mathrm{ub}}}(x)$ when $x \rightarrow 0$ can be derived as

$$
\begin{aligned}
f_{\gamma_{U_{i, l}^{\text {min }}}^{\text {ub }}}^{x \rightarrow 0}(x)= & \frac{\phi_{i}^{N_{i}-N_{R_{l}}+1} x^{N_{i}-N_{R_{l}}}}{\Gamma\left(N_{i}-N_{R_{l}}+1\right)\left(C_{l} \bar{\gamma}_{U, R}\right)^{N_{i}-N_{R_{l}}+1}} \\
& +o\left(x^{N_{i}-N_{R_{l}}+1}\right) \text { for } i \in\{1,2\},
\end{aligned}
$$

where $\bar{\gamma}_{1, l}=\bar{\gamma}_{2, l}=\bar{\gamma}_{U, R_{l}}, \bar{\gamma}_{l, 1}=\bar{\gamma}_{l, 2}=\bar{\gamma}_{R_{l}, U}, \bar{\gamma}_{R_{l}, U}=\nu_{l} \bar{\gamma}_{U, R_{l}}$, and $\bar{\gamma}_{U, R_{l}}=C_{l} \bar{\gamma}_{U, R}$. Further, $\phi_{i}=\left(N_{i}+N_{i^{\prime}}-2 N_{R_{l}}\right) /\left(N_{i^{\prime}}-\right.$ $N_{R_{l}}$ ) for $i \in\{1,2\}, i^{\prime} \in\{1,2\}, i \neq i^{\prime}$. The first order expansion of the CDF of $\gamma_{U_{i, l}^{\min }}^{\mathrm{ub}}$ when $x \rightarrow 0$ can be derived by using (71) as follows:

$$
\begin{aligned}
F_{\gamma_{U_{i, l}}^{\mathrm{min}}}^{x \rightarrow 0}(x)= & \frac{\phi_{i}^{N_{i}-N_{R_{l}}+1}}{\Gamma\left(N_{i}-N_{R_{l}}+2\right) C_{l}^{N_{i}-N_{R_{l}}+1}}\left(\frac{x}{\overline{\gamma_{U, R}}}\right)^{N_{i}-N_{R_{l}}+1} \\
& +o\left(x^{N_{i}-N_{R_{l}}+2}\right) \text { for } i \in\{1,2\}
\end{aligned}
$$

Next, the CDF of $\gamma_{l}^{\mathrm{ub}}=\min \left(\gamma_{U_{1, l}^{\min }}^{\mathrm{ub}}, \gamma_{U_{2, l}^{\min }}^{\mathrm{ub}}\right)$ can be written as

$$
F_{\gamma_{l}^{\mathrm{ub}}}(x)=\sum_{i=1}^{2} F_{\gamma_{U_{i, l}^{\mathrm{min}}}^{\mathrm{ub}}}(x)-\prod_{i=1}^{2} F_{\gamma_{U, l}^{\mathrm{ub}}}(x) .
$$

Then, a polynomial approximation of $F_{\gamma_{l}^{\mathrm{ub}}}(x)$ can be derived by substituting (72) into (73) as (74).

By collecting the lowest powers of $x$ in (74), the first order expansion of $F_{\gamma_{l}^{u b}}(x)$ can be derived as

$$
\begin{aligned}
F_{\gamma_{l}^{\mathrm{ub}}}^{x \rightarrow 0}(x)= & \Theta_{l}\left(\frac{x}{\bar{\gamma}_{U, R}}\right)^{\min \left(N_{1}, N_{2}\right)-N_{R_{l}}+1} \\
& +o\left(\bar{\gamma}_{U, R}^{-\left(\min \left(N_{1}, N_{2}\right)-N_{R_{l}}+2\right)}\right),
\end{aligned}
$$

where $\Theta_{l}$ is defined in (41). The first order expansion of the CDF of $\gamma_{\mathrm{eq}}^{\mathrm{ub}}=\max _{l \in\{1, \cdots, L\}}\left(\gamma_{l}^{\mathrm{lb}}\right)$ can then be derived by first substituting (75) into $F_{\gamma_{\mathrm{eq}}^{\mathrm{ub}}}(x)=\prod_{l=1}^{L}\left(F_{\gamma_{l}^{\mathrm{b}}}(x)\right)$ and then by taking the lowest power of $x$ as shown in (39).

The asymptotic outage upper bound can be derived by substituting the first order expansion of the CDF of $\gamma_{l}^{\mathrm{lb}}$ in [11, Eqn. (35)] into (69) and then taking the lowest power of $x$ as shown in (33).

\section{APPENDIX D}

\section{PROOF OF THE AVERAGE SUM RATE FOR RELAYS WITH DIFFERENT ANTENNA ARRAY SIZES}

In this appendix, the proof of the average sum rate of SS-2 is sketched when the relays are equipped with different number of

\footnotetext{
${ }^{9}$ The first order expansion of $f(x)$ is the single-term polynomial approximation of $f(x)$ consisting the lowest power of $x$ [33].
}

antennas. To begin with, we recall the definition of the average sum rate approximation as follows:

$$
\overline{\mathcal{R}}_{L^{*}} \approx \mathcal{E}_{\gamma_{\mathrm{eq}}}\left\{\left(\max _{l \in\{1, \cdots, L\}}\left(N_{R_{l}}\right)\right) \log \left(1+\gamma_{\mathrm{eq}}\right)\right\},
$$

where $\gamma_{\text {eq }}$ is the equivalent SNR and is given by

$$
\gamma_{\text {eq }}=\min \left(\gamma_{U_{1, L^{*}}^{\min }}, \gamma_{U_{2, L^{*}}^{\min }}\right) \text {. }
$$

Next, an approximation of the CDF of $\gamma_{\mathrm{eq}}$ can be derived by using (19) as follows:

$$
F_{\gamma_{\mathrm{eq}}}(x) \approx 1-\prod_{i=1}^{2}\left(\frac{\Gamma\left(N_{i}-N_{R}^{\max }+1, \frac{\zeta_{i, L^{*} x}}{\alpha_{i, L^{*}}-\beta_{i, L^{*} x}}\right)}{\Gamma\left(N_{i}-N_{R}^{\max }+1\right)}\right),
$$

where $x \leq \min \left(\frac{\alpha_{2, L^{*}}}{\beta_{2, L^{*}}}, \frac{\alpha_{2, L^{*}}}{\beta_{2, L^{*}}}\right)$. Further, $F_{\gamma_{\mathrm{eq}}}(x)=1$ for $x>\min \left(\alpha_{1, L^{*}} / \beta_{1, L^{*}}, \alpha_{2, L^{*}} / \beta_{2, L^{*}}\right)$. Next, by using [21, Eq. (8.352.2)], (78) can be further expanded as

$$
\begin{aligned}
& F_{\gamma_{\mathrm{eq}}}(x) \approx 1-\exp \left(-\frac{\zeta_{1, L^{*}} x}{\alpha_{1, L^{*}}-\beta_{1, L^{*}} x}-\frac{\zeta_{2, L^{*}} x}{\alpha_{2, L^{*}}-\beta_{2, L^{*}} x}\right) \\
& \times \sum_{m=1}^{N_{1}-N_{R}^{\max }} \sum_{n=0}^{N_{2}-N_{R}^{\max }} \frac{1}{m ! n !}\left(\frac{\zeta_{1, L^{*}} x}{\alpha_{1, L^{*}}-\beta_{1, L^{*}} x}\right)^{m}\left(\frac{\zeta_{2, L^{*}} x}{\alpha_{2, L^{*}}-\beta_{2, L^{*}} x}\right)^{n} .
\end{aligned}
$$

The CDF in (79) can further be simplified, whenever both the user nodes are equipped with the same number of antennas (i.e., $N_{1}=N_{2}=N$ ), and all transmit and noise powers at each relay are the same, as follows:

$$
\begin{aligned}
F_{\gamma_{\text {eq }}}(x) \approx & 1-\exp \left(-\frac{2 \zeta_{L^{*}} x}{\alpha_{L^{*}}-\beta_{L^{*}} x}\right) \\
& \times \sum_{m=0}^{N-N_{R}^{\max }} \sum_{n=0}^{N-N_{R}^{\max }} \frac{1}{m ! n !}\left(\frac{\zeta_{L^{*}} x}{\alpha_{L^{*}}-\beta_{L^{*}} x}\right)^{m+n},
\end{aligned}
$$

where $\alpha_{L^{*}}=\alpha_{i, L^{*}}, \beta_{L^{*}}=\beta_{i, L^{*}}$, and $\zeta_{L^{*}}=\zeta_{i, L^{*}}$ for $i \in\{1,2\}$, and $x<\alpha_{L^{*}} / \beta_{L^{*}}$. By differentiating (80), an approximation of the PDF of $\gamma_{\text {eq }}$ can be derived as follows:

$$
\begin{aligned}
f_{\gamma_{\mathrm{eq}}}(x) \approx & \sum_{m=0}^{N-N_{R}^{\max }} \sum_{n=0}^{N-N_{R}^{\max }} \frac{\alpha_{L^{*}} \zeta_{L^{*}}^{m+n} x^{m+n-1}}{m ! n !\left(\alpha_{L^{*}}-\beta_{L^{*}} x\right)^{m+n+1}} \\
& \times\left(\frac{2 \zeta_{L^{*}} x}{\alpha_{L^{*}}-\beta_{L^{*}} x}-(m+n)\right) \exp \left(-\frac{2 \zeta_{L^{*}} x}{\alpha_{L^{*}}-\beta_{L^{*}} x}\right),
\end{aligned}
$$

where $x \leq \alpha_{L^{*}} / \beta_{L^{*}}$. Further, $f_{\gamma_{\mathrm{eq}}}(x)=0$ for $x \geq \alpha_{L^{*}} / \beta_{L^{*}}$. Next, an approximation of the ergodic sum rate of SS-2 can be derived by averaging the sum rate in (57) over the PDF of $\gamma_{\mathrm{eq}}$ in (81) as

$$
\overline{\mathcal{R}}_{L^{*}} \approx \mathcal{E}\left\{\mathcal{R}_{L^{*}}\right\}=\frac{N_{R}^{\max }}{\ln (2)} \int_{0}^{\infty} \ln (1+x) f_{\gamma_{\mathrm{eq}}}(x) \mathrm{d} x .
$$


By substituting (81) into (82), the ergodic sum rate lower bound can be written in an integral form as follows:

$$
\overline{\mathcal{R}}_{L^{*}} \approx \frac{N_{R}^{\max }}{\ln (2)} \sum_{m=0}^{N-N_{R}^{\max }} \sum_{n=0}^{N-N_{R}^{\max }} \frac{1}{m ! n !}\left(\mathbb{I}_{1}-\mathbb{I}_{2}\right),
$$

where $\mathbb{I}_{1}$ and $\mathbb{I}_{2}$ can be defined as follows:

$$
\begin{aligned}
\mathbb{I}_{1}= & 2 \alpha_{L^{*}} \zeta_{L^{*}}^{m+n+1} \int_{0}^{\frac{\alpha_{L^{*}}}{\beta_{L^{*}}}} \frac{x^{m+n}}{\left(\alpha_{L^{*}}-\beta_{L^{*}} x\right)^{m+n+2}} \\
& \times \exp \left(-\frac{2 \zeta_{L^{*}} x}{\alpha_{L^{*}}-\beta_{L^{*} x}}\right) \ln (1+x) \mathrm{d} x, \\
\mathbb{I}_{2}= & (m+n) \alpha_{L^{*}} \zeta_{L^{*}}^{m+n} \int_{0}^{\frac{\alpha_{L^{*}}}{\beta_{L^{*}}}} \frac{x^{m+n-1}}{\left(\alpha_{L^{*}}-\beta_{L^{*}} x\right)^{m+n+1}} \\
& \times \exp \left(-\frac{2 \zeta_{L^{*}} x}{\alpha_{L^{*}}-\beta_{L^{*}} x}\right) \ln (1+x) \mathrm{d} x .
\end{aligned}
$$

By substituting the dummy variable $t=2 \zeta_{L^{*}} x /\left(\alpha_{L^{*}}-\beta_{L^{*}} x\right)$ into (83b) and (83c), the integrals $\mathbb{I}_{1}$ and $\mathbb{I}_{2}$ can be simplified as

$$
\begin{aligned}
& \mathbb{I}_{1}=\frac{1}{2^{m+n}} \int_{0}^{\infty} t^{m+n} \mathrm{e}^{-t} \ln \left(\frac{2 \zeta_{L^{*}}+\left(\alpha_{L^{*}}+\beta_{L^{*}}\right) t}{2 \zeta_{L^{*}}+\beta_{L^{*}} t}\right) \mathrm{d} t \\
& \mathbb{I}_{2}=\frac{m+n}{2^{m+n}} \int_{0}^{\infty} t^{m+n-1} \mathrm{e}^{-t} \ln \left(\frac{2 \zeta_{L^{*}}+\left(\alpha_{L^{*}}+\beta_{L^{*}}\right) t}{2 \zeta_{L^{*}}+\beta_{L^{*}} t}\right) \mathrm{d} t .
\end{aligned}
$$

Next, $\mathbb{I}_{1}$ and $\mathbb{I}_{2}$ in (84a) and (84b), respectively, can be solved in closed-form as follows:

$$
\begin{aligned}
\mathbb{I}_{1}= & \frac{1}{2^{m+n}}\left(\mathbb{J}\left(m+n, 2 \zeta_{L^{*}}, \alpha_{L^{*}}+\beta_{L^{*}}\right)\right. \\
& \left.-\mathbb{J}\left(m+n, 2 \zeta_{L^{*}}, \beta_{L^{*}}\right)\right), \\
\mathbb{I}_{2}= & \frac{m+n}{2^{m+n}}\left(\mathbb{J}\left(m+n-1,2 \zeta_{L^{*}}, \alpha_{L^{*}}+\beta_{L^{*}}\right)\right. \\
& \left.\quad-\mathbb{J}_{2}\left(m+n-1,2 \zeta_{L^{*}}, \beta_{L^{*}}\right)\right) .
\end{aligned}
$$

where the function $\mathbb{J}(x, y, z)$ is defined in (60). By substituting (85a) and (85b) into (83a), an approximation of the ergodic sum rate of SS-2 can be derived in closed-form as in (59).

\section{APPENDIX E}

PROOF OF THE AVERAGE SUM RATE FOR RELAYS WITH SAME

\section{ANTENNA ARRAY SIZE}

In this appendix, the proof of the average sum rate approximation for the case of equal number of antennas at each relay is sketched. To begin with, the effective SNR (77) can be alternatively approximated as follows:

$$
\gamma_{\mathrm{eq}} \approx \gamma_{\mathrm{eq} 1}=\max _{l \in\{1, \cdots, L\}}\left(\min \left(\gamma_{U_{1, l}^{\min }}^{\mathrm{ub}}, \gamma_{U_{2, l}^{\min }}^{\mathrm{ub}}\right)\right)
$$

where $\gamma_{U_{i, l}^{\min }}^{\mathrm{ub}}$ for $i \in\{1,2\}$ is defined in (18). The CDF of $\gamma_{\text {eq }}$ can be derived as follows:

$$
F_{\gamma_{\mathrm{eq} 1}}(x)=\prod_{l=1}^{L}\left(1-\bar{F}_{\gamma_{U_{1, l}^{\min }}^{\mathrm{ub}}}(x) \bar{F}_{\gamma_{U_{2, l}^{\min }}^{\mathrm{ub}}}(x)\right) .
$$

where $\bar{F}_{\gamma_{U_{i, l}^{\min }}^{\mathrm{ub}}}(x)$ is the complimentary CDF of $\gamma_{U_{i, l}^{\min }}^{\mathrm{ub}}$. By using (19), $F_{\gamma_{\mathrm{eq}}}(x)$ in (87) can be expanded as

$$
F_{\gamma_{\mathrm{eq} 1}}(x)=\prod_{l=1}^{L}\left(1-\prod_{i=1}^{2}\left(\frac{\Gamma\left(N_{i}-N_{R}+1, \frac{\zeta_{i, l} x}{\alpha_{i, l}-\beta_{i, l} x}\right)}{\Gamma\left(N_{i}-N_{R}+1\right)}\right)\right) \text {. }
$$

By using [21, Eq. (8.352.2)], (88) can be further expanded as

$$
\begin{aligned}
& F_{\gamma_{\mathrm{eq} 1}}(x)=\prod_{l=1}^{L}\left(1-\exp \left(-\frac{\zeta_{1, l} x}{\alpha_{1, l}-\beta_{1, l} x}-\frac{\zeta_{2, l} x}{\alpha_{2, l}-\beta_{2, l} x}\right)\right. \\
& \left.\quad \times \sum_{m=1}^{N_{1}-N_{R}} \sum_{n=0}^{N_{2}-N_{R}} \frac{1}{m ! n !}\left(\frac{\zeta_{1, l} x}{\alpha_{1, l}-\beta_{1, l} x}\right)^{m}\left(\frac{\zeta_{2, l} x}{\alpha_{2, l}-\beta_{2, l} x}\right)^{n}\right),
\end{aligned}
$$

where $x<\min \left(\alpha_{1, l} / \beta_{1, l}, \alpha_{2, l} / \beta_{2, l}\right)$, and $F_{\gamma_{\mathrm{eq}}}(x)=1$ for $x \geq \min \left(\alpha_{1, l} / \beta_{1, l}, \alpha_{2, l} / \beta_{2, l}\right)$. The CDF in (89) can further be simplified whenever both the user nodes are equipped with the same number of antennas (i.e., $N_{1}=N_{2}=N$ ) as follows:

$$
\begin{aligned}
F_{\gamma_{\mathrm{eq} 1}}(x)= & \prod_{l=1}^{L}\left(1-\exp \left(-\frac{2 \zeta_{l} x}{\alpha_{l}-\beta_{l} x}\right)\right. \\
& \left.\times \sum_{m=1}^{N-N_{R}} \sum_{n=0}^{N-N_{R}} \frac{1}{m ! n !}\left(\frac{\zeta_{l} x}{\alpha_{l}-\beta_{l} x}\right)^{m+n}\right),
\end{aligned}
$$

where $x<\alpha_{l} / \beta_{l}, \alpha_{l}=\alpha_{1, l}=\alpha_{2, l}$, and $\beta_{l}=\beta_{1, l}=\beta_{2, l}$. Further, $F_{\gamma_{\mathrm{eq}}}(x)=1$ for $x \geq \alpha_{l} / \beta_{l}$. The close-form derivation of the ergodic sum rate of SS-2 by using (90) appears mathematical intractable. Nevertheless, in the case of all transmit and noise powers at all the relays are the same, (90) can be written as follows:

$$
\begin{aligned}
F_{\gamma_{\mathrm{eq}}}(x)= & \left(1-\exp \left(-\frac{2 \zeta x}{\alpha-\beta x}\right)\right. \\
& \left.\times \sum_{m=0}^{N-N_{R}} \sum_{n=0}^{N-N_{R}} \frac{1}{m ! n !}\left(\frac{\zeta x}{\alpha-\beta x}\right)^{m+n}\right)^{L},
\end{aligned}
$$

where $\alpha_{l}=\alpha, \zeta_{l}=\zeta, \beta_{l}=\beta$ for $l \in\{1,2, \ldots L\}$, and $x<\alpha / \beta$. By using the binomial expansion, (80) can be further expanded as follows:

$$
\begin{aligned}
F_{\gamma_{\mathrm{eq} 1}}(x)= & \sum_{l=0}^{L}(-1)^{l}\left(\begin{array}{l}
L \\
l
\end{array}\right) \exp \left(-\frac{2 l \zeta x}{\alpha-\beta x}\right) \\
& \times\left(\sum_{m=0}^{N-N_{R}} \frac{1}{m !}\left(\frac{\zeta x}{\alpha-\beta x}\right)^{m}\right)^{2 l} .
\end{aligned}
$$

Next, by using [34, Eqn. (44)] and [35, Eqn. (6)], the CDF of $\gamma_{\mathrm{eq}}$ can be finally written as

$$
\begin{aligned}
F_{\gamma_{\mathrm{eq} 1}}(x)= & \sum_{l=0}^{L} \sum_{k=0}^{2 l\left(N-N_{R}\right)}(-1)^{l}\left(\begin{array}{c}
L \\
l
\end{array}\right) \beta_{k, 2 l, N-N_{R}+1}\left(\frac{\zeta x}{\alpha-\beta x}\right)^{k} \\
& \times \exp \left(-\frac{2 l \zeta x}{\alpha-\beta x}\right) \text { for } x<\frac{\alpha}{\beta}
\end{aligned}
$$

where the multinomial coefficient $\beta_{k, 2 l, N-N_{R}+1}$ is defined in (62). By differentiating (93), the PDF of $\gamma_{\mathrm{eq}}$ can be derived as follows:

$$
\begin{aligned}
f_{\gamma_{\mathrm{eq}_{1}}}(x)= & \sum_{l=1}^{L} \sum_{k=0}^{2 l\left(N-N_{R}\right)}(-1)^{l}\left(\begin{array}{c}
L \\
l
\end{array}\right) \beta_{k, 2 l, N-N_{R}+1} \\
& \times\left(k-\frac{2 l \zeta x}{\alpha-\beta x}\right) \frac{\alpha \zeta^{k} x^{k-1}}{(\alpha-\beta x)^{k+1}} \exp \left(-\frac{2 l \zeta x}{\alpha-\beta x}\right),
\end{aligned}
$$


where $x<\alpha / \beta$. Further, $f_{\gamma_{\mathrm{eq}}}(x)=0$ for $x \geq \alpha / \beta$. Next, the approximation of the ergodic sum rate of SS- 2 can be derived by averaging the sum rate in (57) over the PDF of $\gamma_{\mathrm{eq}}$ in (94) as

$$
\overline{\mathcal{R}}_{L^{*}} \approx \mathcal{E}\left\{\mathcal{R}_{L^{*}}\right\}=\frac{N_{R}}{\ln (2)} \int_{0}^{\infty} \ln (1+x) f_{\gamma_{\mathrm{eq}_{1}}}(x) \mathrm{d} x .
$$

Using similar techniques to those used in (59), a tight approximation to the ergodic sum rate of SS-2, whenever all relays are equipped with the same number of antennas, can be derived as in (61).

\section{REFERENCES}

[1] S. Silva, G. Amarasuriya, C. Tellambura, and M. Ardakani, "Relay selection for MIMO Two-Way relay networks with spatial multiplexing," in IEEE ICC 2015 - Workshop on Cooperative and Cognitive Networks (CoCoNet) (ICC'15 - Workshops 16), London, United Kingdom, Jun. 2015, pp. $943-$ 948.

[2] A. Bletsas, A. Khisti, D. P. Reed, and A. Lippman, "A simple cooperative diversity method based on network path selection," IEEE J. Sel. Areas Commun., vol. 24, no. 3, pp. 659-672, Mar. 2006.

[3] Y. Jing and H. Jafarkhani, "Single and multiple relay selection schemes and their achievable diversity orders," IEEE Trans. Wireless Commun., vol. 8, no. 3, pp. 1414-1423, Mar. 2009

[4] Y. Zhao, R. Adve, and T. J. Lim, "Improving amplify-and-forward relay networks: optimal power allocation versus selection," IEEE Trans. Wireless Commun., vol. 6, no. 8, pp. 3114-3123, Aug. 2007.

[5] B. Rankov and A. Wittneben, "Spectral efficient protocols for half-duplex fading relay channels," IEEE J. Sel. Areas Commun., vol. 25, no. 2, pp. 379-389, Feb. 2007

[6] G. Amarasuriya, C. Tellambura, and M. Ardakani, "Two-way amplifyand-forward multiple-input multiple-output relay networks with antenna selection," IEEE J. Sel. Areas Commun., vol. 30, no. 8, pp. 1513-1529, Sep. 2012

[7] K. Loa et al., "IMT-advanced relay standards," IEEE Commun. Mag., vol. 48, no. 8, pp. 40-48, Aug. 2010

[8] Y. Yang, H. Hu, J. Xu, and G. Mao, "Relay technologies for WiMax and LTE-advanced mobile systems," IEEE Commun. Mag., vol. 47, no. 10, pp. 100-105, Oct. 2009

[9] A. Paulraj, R. Nabar, and D. Gore, Introduction to space-time wireless communications, 1st ed. Cambridge, UK ; New York, NY : Cambridge University Press, 2003.

[10] L. Zheng and D. Tse, "Diversity and multiplexing: a fundamental tradeoff in multiple-antenna channels," IEEE Trans. Inf. Theory, vol. 49, no. 5, pp. 1073-1096, May 2003.

[11] G. Amarasuriya, C. Tellambura, and M. Ardakani, "Performance analysis of zero-forcing for two-way MIMO AF relay networks," IEEE Wireless Commun. Lett., vol. 1, no. 2, pp. 53-56, Apr. 2012.

[12] _ - "Joint beamforming and antenna selection for two-way amplify-andforward MIMO relay networks," in IEEE Int. Conf. on Commun. (ICC), Jun. 2012, pp. 4829-4834.

[13] S. Atapattu, Y. Jing, H. Jiang, and C. Tellambura, "Relay selection schemes and performance analysis approximations for two-way networks," IEEE Trans. Commun., vol. 61, no. 3, pp. 987-998, Mar. 2013.

[14] Y. Li, R. H. Y. Louie, and B. Vucetic, "Relay selection with network coding in two-way relay channels," IEEE Trans. Veh. Technol., vol. 59, no. 9, pp. 4489-4499, 2010.

[15] R. Zhang, Y.-C. Liang, C. C. Chai, and S. Cui, "Optimal beamforming for two-way multi-antenna relay channel with analogue network coding," IEEE J. Sel. Areas Commun., vol. 27, no. 5, pp. 699-712, Jun. 2009.

[16] H. Cui, M. Ma, L. Song, and B. Jiao, "Relay selection for two-way full duplex relay networks with amplify-and-forward protocol," IEEE Trans. Wireless Commun., vol. 13, no. 7, pp. 3768-3777, Jul. 2014.

[17] I. Krikidis, "Relay selection for two-way relay channels with MABC DF: A diversity perspective," IEEE Trans. Veh. Technol., vol. 59, no. 9, pp. 46204628, Nov. 2010
[18] Q. Zhou, Y. Li, F. Lau, and B. Vucetic, "Decode-and-forward two-way relaying with network coding and opportunistic relay selection," IEEE Trans. Commun., vol. 58, no. 11, pp. 3070-3076, Nov. 2010.

[19] T. Oechtering and H. Boche, "Bidirectional regenerative half-duplex relaying using relay selection," IEEE Trans. Wireless Commun., vol. 7, no. 5, pp. 1879-1888, May 2008.

[20] L. Song, G. Hong, B. Jiao, and M. Debbah, "Joint relay selection and analog network coding using differential modulation in two-way relay channels," IEEE Trans. Veh. Technol., vol. 59, no. 6, pp. 2932-2939, Jul. 2010.

[21] I. Gradshteyn and I. Ryzhik, Table of integrals, Series, and Products, 7th ed. Academic Press, 2007.

[22] R. H. Y. Louie, Y. Li, and B. Vucetic, "Practical physical layer network coding for two-way relay channels: performance analysis and comparison," IEEE Trans. Wireless Commun., vol. 9, pp. 764-777, Feb. 2010.

[23] A. Sulyman et al., "Radio propagation path loss models for 5G cellular networks in the $28 \mathrm{GHz}$ and $38 \mathrm{GHz}$ millimeter-wave bands," IEEE Commun. Mag., vol. 52, no. 9, pp. 78-86, Sep. 2014.

[24] A. Swindlehurst, E. Ayanoglu, P. Heydari, and F. Capolino, "Millimeterwave massive MIMO: the next wireless revolution?" IEEE Commun. Mag., vol. 52, no. 9, pp. 56-62, Sep. 2014.

[25] J. Heath, R.W., S. Sandhu, and A. Paulraj, "Antenna selection for spatial multiplexing systems with linear receivers," IEEE Commun. Lett., vol. 5, no. 4, pp. 142-144, Apr. 2001.

[26] M. Antonia and S. Verdu, Random Matrix Theory and Wireless Communications, 1st ed., ser. Foundations and Trends in Communications and Information Theory. Now publishers inc, 2004, no. 1.

[27] L. G. Ordóñez, "Performance limits of spatial multiplexing MIMO systems," $\mathrm{Ph} . \mathrm{D}$. dissertation, Technical University of Catalonia (UPC), Barcelona, Spain, Mar. 2009

[28] D. Gore, J. Heath, R.W., and A. Paulraj, "On performance of the zero forcing receiver in presence of transmit correlation," in IEEE Intl. Symp. on Inf. Theory (ISIT), Lausanne, Switzerland, Jul. 2002, pp. 1-2.

[29] E. F. Beckenbach and R. E. Bellman, Inequalities, 1st ed. Springer Verlag, 1965.

[30] A. R. Amir-Moez and G. E. Johnston, "On the product of diagonal elements of a positive matrix," Mathematics Magazine of Mathematical Association of America, vol. 42, no. 1, pp. 24-26, Jan. 1969.

[31] D. N. C. Tse, P. Viswanath, and L. Zheng, "Diversity-multiplexing tradeoff in multiple-access channels," IEEE Trans. Inf. Theory, vol. 50, no. 9, pp. 1859-1874, Sep. 2004

[32] A. Papoulis and S. U. Pillai, Probability, Random Variables and Stochastic Processes, 4th ed. McGraw-Hill, Inc., NY, 2002.

[33] Z. Wang and G. B. Giannakis, "A simple and general parameterization quantifying performance in fading channels," IEEE Trans. Commun., vol. 51, no. 8, pp. 1389-1398, Aug. 2003.

[34] A. Annamalai and C. Tellambura, "Error rates for Nakagami- $m$ fading multichannel reception of binary and M-ary signals," IEEE Trans. Commun., vol. 49 , no. 1 , pp. 58-68, Jan. 2001.

[35] G. Amarasuriya, C. Tellambura, and M. Ardakani, "Transmit antenna selection strategies for cooperative MIMO AF relay networks," in IEEE Global Telecommun. Conf. (GLOBECOM 2010), Dec. 2010, pp. 1-5.

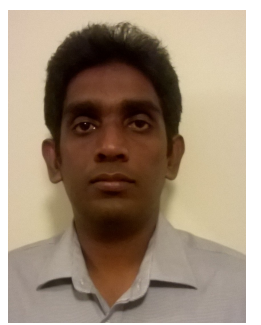

Shashindra Silva (S'10) received the B.Sc. degree in Electronic and Telecommunication Engineering from the University of Moratuwa, Moratuwa, Sri Lanka, in 2013 and the M.Sc. degree in Electrical and Computer Engineering from the University of Alberta, AB, Canada, in 2015. He is currently working towards the Ph.D. degree at the Electrical and Computer Engineering Department, University of Alberta, AB, Canada. His current research interests include massive MIMO and cooperative MIMO relay networks. 


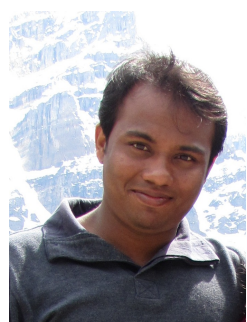

Gayan Amarasuriya (S09-M13) received the B.Sc. degree in engineering with first-class honours in 2006 from the Department of Electronics and Telecommunications Engineering in the University of Moratuwa, Sri Lanka. He received the Ph.D. degrees in electrical engineering from the Department of Electrical and Computer Engineering in the University of Alberta, Canada in 2013. He is currently a postdoctoral research fellow at the Department of Electrical Engineering in Princeton University, Princeton, NJ, USA. His current research interests include massive MIMO, millimeterwave cellular networks, wireless energy harvesting, and cooperative MIMO relay networks.

Dr. Amarasuriya was awarded the exemplary reviewer certificates for IEEE Communication Letters in 2011 and 2012, and for IEEE Wireless Communication Letters in 2013 by the IEEE communication society. In recognition of Dr Amarasuriya's academic, research and scholarly achievements, he was awarded four major awards: (i) Natural Sciences and Engineering Research Council of Canada (NSERC) postdoctoral fellowship, (ii) Izaak Walton Killam Memorial Scholarship, (iii) Alberta Innovates-Technology Future Graduate Student Scholarship in Information and Communications Technology, and (iv) Andrew Stewart Memorial Graduate Prize.

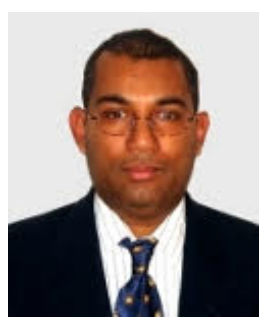

Chintha Tellambura (F11) received the B.Sc. degree (with first-class honor) from the University of Moratuwa, Sri Lanka, in 1986, the M.Sc. degree in Electronics from the University of London, United Kingdom, in 1988, and the Ph.D. degree in Electrical Engineering from the University of Victoria, Canada, in 1993. He was a Postdoctoral Research Fellow with the University of Victoria (1993-1994) and the University of Bradford (1995-1996). He was with Monash University, Australia, from 1997 to 2002. Presently, he is a Professor with the Department of Electrical and Computer Engineering, University of Alberta. His current research interests include the design, modelling and analysis of cognitive radio networks, heterogeneous cellular networks and multiple-antenna wireless networks.

Prof. Tellambura served as an editor for both IEEE Transactions on Communications (1999-2011) and IEEE Transactions on Wireless Communications (2001-2007) and was the Area Editor for Wireless Communications Systems and Theory in the IEEE Transactions on Wireless Communications during 2007-2012. Prof. Tellambura and co-authors received the Communication Theory Symposium best paper award in the 2012 IEEE International Conference on Communications, Ottawa, Canada. He is the winner of the prestigious McCalla Professorship and the Killam Annual Professorship from the University of Alberta. Prof. Tellambura has authored or coauthored over 480 journal and conference publications with total citations more than 10,000 and an h-index of 52 (Google Scholar).

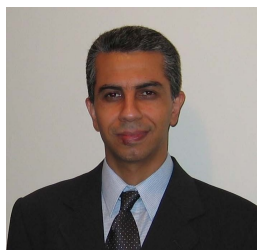

Masoud Ardakani (M'04-SM'09) received the B.Sc. degree from Isfahan University of Technology in 1994 , the M.Sc. degree from Tehran University in 1997, and the Ph.D. degree from the University of Toronto, Canada, in 2004, all in electrical engineering. He was a Postdoctoral fellow at the University of Toronto from 2004 to 2005 . He is currently a Professor of Electrical and Computer Engineering at the University of Alberta, Canada. His research interests are in the general area of digital communications. Dr. Ardakani serves as an Associate Editor for the IEEE TRANSACTIONS ON COMMUNICATIONS and has served as an Associate Editor for the IEEE WIRELESS COMMUNICATIONS and as a senior editor for the IEEE COMMUNICATION LETTERS. 